\title{
HARNESSING HUMAN STEM CELLS FOR THE TREATMENT OF GLIOBLASTOMA TWENTY YEAR PERSPECTIVE \\ Review of preclinical and clinical studies: $2000-2020$
}

Anda-Alexandra Calinescu ${ }^{1 *}$, McKenzie C. Kauss ${ }^{1,2}$, Zain Sultan $^{1,3}$, Wajd N. Al-Holou${ }^{1}$, Sue K. O’Shea ${ }^{4}$

1. Department of Neurosurgery, University of Michigan Medical School

2. College of Literature Science and Arts, University of Michigan

3. College of Engineering, University of Michigan

4. Department of Cell and Developmental Biology, University of Michigan Medical School

* corresponding author (calinesq@med.umich.edu)

\section{ABSTRACT}

The potential of Neural Stem Cells (NSCs) to provide therapeutic benefit for a variety of neurological disorders, including brain malignancies, has been long recognized and has inspired many scientists to design, test and successfully demonstrate that NSCs are efficient and effective therapeutic agents. Glioblastoma, the deadliest form of primary brain tumor, despite extensive and sustained efforts to find better therapies, remains a disease without cure, with a median survival after diagnosis of less than two years. Treatment resistance in glioblastoma is in large part attributed to limitations in the delivery and distribution of therapeutic agents administered either systemically or directly into the tumor due to the highly invasive nature of this cancer and its abnormal intratumoral vasculature. Stem Cells (SCs) have an innate tumor-tropic migratory behavior, can be modified to deliver a variety of therapeutic agents and efficiently distribute their cargo into brain tumors, pursuing invading streams of tumor cells, deep into the brain parenchyma. Over the last twenty years, numerous preclinical trials have demonstrated the feasibility and efficacy of SCs as antiglioma agents, leading to the development of trials to test these therapies in the clinic. In this review we present and analyze these studies and discuss mechanisms underlying their beneficial effect, highlighting experimental progress, limitations and the emergence of promising new therapeutic avenues. We hope to increase awareness of the advantages of using SCs for the treatment of glioblastoma and inspire further studies that will lead to accelerated implementation of effective therapies.

Keywords: Glioblastoma, Neural Stem Cells, Mesenchymal Stem Cells, Stem Cell Therapy, Enzyme/Prodrug Therapy, Oncolytic Virotherapy, Nanoparticles, TRAIL, Cytokine therapy 


\section{SECTIONS}

1. THE BEGINNINGS OF THERAPEUTIC STEM CELLS

2. ORIGINS OF STEM CELLS USED FOR THE TREATMENT OF BRAIN TUMORS

3. THERAPEUTIC STRATEGIES AND THEIR MECHANISMS OF ACTION

3.1 STEM CELLS IN ENZYME/PRODRUG STRATEGIES

3.2 STEM CELLS CARRYING PRO-APOPTOTIC MOLECULES

3.3 STEM CELLS AND ONCOLYTIC VIROTHERAPY

3.4 THERAPEUTIC CYTOKINES, ANTIBODIES AND TOXINS DELIVERED BY STEM CELLS

3.5 STEM CELL TRAFFICKING OF THERAPEUTIC NANOPARTICLES

4. OUTLOOK FOR THE FUTURE

\section{THE BEGINNINGS OF THERAPEUTIC STEM CELLS}

The existence of stem cells, as proliferating, specialized cells present in the periventricular spaces of the embryonic brain, postulated to give rise to terminally differentiated neurons and glia, was first recognized by the Swiss neurologist Wilhelm His $(1874)^{1,2}$. The presence, properties and potential of these cells has fascinated the neuroscience community ever since. Studies in the mid-20 th century established them as the source of both neurons and macroglia in the brain. Toward the end of the $20^{\text {th }}$ century, when numerous elegant lineage-tracing studies demonstrated the ability of NSCs to become and potentially replenish any cell type in the CNS, neuroscientists agreed to name these cells "Neural Stem Cells" (NSCs) ${ }^{3-6}$. Recognition of the therapeutic potential of NSCs for various neuro-degenerative diseases and traumatic injuries of the CNS was practically instantaneous. Early preclinical studies have demonstrated the potential use of NSCs in lysosomal storage diseases ${ }^{7}$, Huntington's disease ${ }^{8}$, Parkinson's disease ${ }^{9,10}$, Multiple Sclerosis ${ }^{11}$, Amyotrophic Lateral Sclerosis ${ }^{12}$ and Spinal Cord Injury ${ }^{13}$. Today, more than 40 clinical trials are testing the use of Neural Stem Cells for the treatment of a variety of neurological disorders, including glioblastoma (clinicaltrials.gov). However, translation of preclinical findings into approved therapies has been hindered by several barriers, including ethical concerns related to the use of fetal derived cells, availability of appropriate cells developed into approved "off the shelf" investigational drugs, limitations in manufacturing, quality control and cost of autologous stem cells, drawbacks from adverse consequences encountered in unapproved Stem Cell interventions ${ }^{14}$ and the enormous cost and effort required to assemble a functional collaboration of scientists, clinicians, industry, regulatory bodies and funding agencies to promote and expedite approval and implementation of clinical trials to test the efficacy of SC based therapies ${ }^{15}$.

Malignant gliomas are the most common primary brain tumors, with an incidence of $~ 6 / 100,000$ (2010$2014)^{16}$. The majority of gliomas (61.5\%) are glioblastomas (GBM), the most aggressive form, for which no cure exists. Under standard of care (SOC), which includes: maximal safe tumor resection followed by radiation and chemotherapy ${ }^{17}$, the median life expectancy for patients diagnosed with GBM remains dismal: 15-17 months ${ }^{16}$. Following SOC, tumors invariably recur and progress to an increasingly more aggressive form. Recurrence of GBM is attributed to many factors: (1) the heterogeneous makeup of the tumors, making them difficult to target with single agents; (2) the blood-brain-barrier (BBB) alongside abnormal intra-tumoral 
vascularization, that prevent drug distribution; (3) the highly invasive and infiltrative nature of the disease, with glioma cells penetrating the brain parenchyma at great distance from the bulk of the tumor; (4) the presence of glioma stem cells (GSCs), also referred to as brain tumor initiating cells (BTIC), or Recurrence Initiating Stem Cells ${ }^{18}$ that are resistant to chemo- and radiotherapy and can initiate tumor formation, and (5) an immunosuppressive tumor microenvironment permissive for tumor growth. GSCs share many characteristics with NSCs including high proliferative potential, association with blood vessels, telomerase activity, diverse progeny and similar gene expression profiles including Nestin, CD133, Olig2, Sox2, Sox4, Integrin- $\alpha 6$, CD15, L1CAM, BMI1, A2B5, CD44 and others ${ }^{19-23}$.

The idea that at the origin of GBM lies a Neural Stem Cell gone awry has long been suggested, but never entirely proven ${ }^{24}$. There exists nonetheless "circumstantial" evidence that this may be the case, at least in a subset of tumors. For example, it was demonstrated that glioblastomas that are in contact with the subventricular zone (SVZ) and reach the cortex, were more likely to be multifocal at initial diagnosis and/or to progress to multifocal disease at recurrence ${ }^{25}$, suggesting a mechanism by which transformed cells in the SVZ (either NSCs or their progeny) migrate out towards the cortex, similar to the inside-out migration of neural progenitor cells (NPCs) guided by radial glia during cortical development ${ }^{26}$. Our understanding of what constitutes a Neural Stem Cell, namely a cell that can self-renew and generate both differentiated neurons and glia has evolved with studies demonstrating that some glial cells such as the radial glia (RG) of the ventricular zone (VZ) and SVZ during development ${ }^{27}$ as well as specialized astrocytes (B cells) and radial astrocytes present in neurogenic zones of the adult (the SVZ and the Subgranular Zone (SGZ) of the hippocampus) give rise to proliferating precursors, named also Intermediate Progenitor Cells (IPCs) that can generate both Neural Precursor Cells (NPCs) and glial precursors: Oligodendrocyte Precursor Cells (OPCs) and astrocytes ${ }^{28}$. NPCs are also multipotent progenitors that proliferate but have a more limited capacity of self-renewal and can differentiate into at least two different cell lineages ${ }^{29}$. Importantly, when cultured in vitro using the Neurosphere Assay in serum free media supplemented with EGF and FGF, neurospheres derived from one NSC are a heterogeneous population of stem and progenitor cells, usually referred to as Neural Stem and Progenitor Cells (most commonly abbreviated as NPCs), that can give rise to neurons, oligodendrocytes and astrocytes ${ }^{30-}$ 32.

Interestingly, a recent study identified, using single cell RNA sequencing (scRNA-Seq) of glioblastoma samples collected from 11 patients, a subpopulation of $R G$ with high invasive potential, that also harbored GSC genetic signature and displayed the mitotic somatic translocation phenotype characteristic of developmental $\mathrm{RG}^{33}$. Whether this reflects a potential origin of glioblastomas from arrested embryonic tissue that was reactivated during carcinogenesis is difficult to say, nonetheless, these findings support the idea that malignancy makes use of developmental mechanisms to promote growth and invasion. This study, as well as other recent studies using the powerful scRNA-Seq technology ${ }^{34-37}$ highlights the enormously heterogeneous nature of glioblastomas and the widespread expression of GSC markers in the majority of tumor cell types described. 
The CXCL12 chemokine and its cognate signaling receptor CXCR4, originally identified as a homeostatic chemokine/receptor complex that regulates hematopoietic stem cell (HSC) trafficking, activates one of the main signaling pathways that drives NPC migration in the developing $\mathrm{CNS}^{38}$, and also guides migration of GSCs ${ }^{39}$. CXCL12 is highly expressed in the SVZ ${ }^{39}$ and in the bone marrow HSC niche where it promotes migration of primitive hematopoietic cells ${ }^{40}$. Production of CXCL12 is increased inside tumors in response to hypoxia and irradiation ${ }^{41}$. High levels of CXCL12 are also found in pseudopallisades ${ }^{42}$, a hallmark histological feature of WHO grade IV GBM, regions that also harbor GSCs ${ }^{43}$. GSCs and NSCs thus have shared migratory behavior, due at least in part to their responsiveness to CXCL12 chemotaxis.

Transplanted rodent NSCs exhibit an intrinsic migratory behavior, following developmental pathways $^{44,45}$. Human NSCs, generated from the periventricular telencephalic region of a 15-week old human fetus and immortalized by retroviral transduction with v-myc, were injected into the lateral ventricles of newborn mice and their distribution into the brain was analyzed over time, for up to 5 weeks. As early as 24 hours after injection, the human NSCs had joined migrating endogenous mouse neuroblasts in the rostral migratory stream. At three weeks these cells were found in the olfactory bulb, differentiated into neurons ${ }^{46}$. Implanted human NSCs were also found to differentiate into oligodendrocytes, astrocytes or cerebellar granule cells depending on the site of transplantation, demonstrating their multipotency. Interestingly, it was observed that, while the immortalized human NSCs proliferated well in vitro, once transplanted into mouse brains, expression of v-myc was downregulated and the cells stopped dividing ${ }^{46}$. The underlying cause of this behavior is not known, but is assumed to be a consequence of normal developmental mechanisms that induce mitotic arrest during differentiation ${ }^{46}$. Similar observations were made with mouse immortalized $\mathrm{NSCs}^{47,48}$.

Considering the pressing need to find better strategies for intratumoral distribution of therapeutic agents, scientists tested immortalized NSCs in a mouse model of glioblastoma ${ }^{49}$. It was elegantly demonstrated that when murine NSCs (C17.2 cells that were derived from the cerebellum of 4 day old mice and immortalized with $v-\mathrm{myc}^{50}$ ) were injected into the tumor or at a distance from the tumor (in the contralateral hemisphere, intraventricular, or intravenously), distributed widely throughout the tumor and followed invasive streams of glioma cells deep into the brain parenchyma, albeit with lower efficacy following systemic administration ${ }^{49}$. This tumor tropic migratory behavior was not altered when cells were transduced to express a therapeutic enzyme, proof of principle that NSCs have the potential to deliver therapeutic agents in glioblastoma.

\section{ORIGINS OF STEM CELLS USED FOR THE TREATMENT OF BRAIN TUMORS}

In addition to NSCs, Mesenchymal Stem Cells (MSCs) as well as induced NSCs (iNSCs), derived from pluripotent Stem Cells or transdifferentiated from somatic cells, have been shown to display tumor-tropic behavior and distribute extensively in intracranial gliomas. Many preclinical trials have tested these cells for 
their ability to distribute throughout the tumor and deliver a variety of therapeutic agents: bioactive proteins, viruses, cytokines, antibodies, toxins or nanoparticles. The majority of these studies tested the use of human SCs and the rest, rodent SCs. Immortalized human NSCs were most often used, followed by MSCs derived either from bone marrow (BM), adipose tissue, umbilical cord or amniotic fluid, and one study used iNSCs transdifferentiated from fibroblasts. Rodent SCs were employed similarly (SupplementaryTable 1).

Interestingly, all but one study with human NSCs used cells that were obtained from fetal human brain and immortalized with the v-myc oncogene, for easy in vitro propagation and to prevent terminal differentiation when exposed to serum ${ }^{51}$.

One line, the HB1.F3 cell line was derived from the telencephalon of a human female 15-week gestation fetus, initially propagated on poly-lysine-coated tissue culture plates in DMEM supplemented with $5 \%$ horse serum and transduced with a replication incompetent retroviral vector encoding v-myc. Successfully transduced cells were plated at clonal density and several clones were selected, including HB1.F3 ${ }^{52}$. The HB1.F3.CD cell line was derived from HB1.F3 cells that was transduced to express cytosine deaminase(CD) ${ }^{49}$, and subsequently extensively characterized and FDA approved for use in clinical trials ${ }^{53}$. The HB1.F3.CD cell line is easily propagated in culture in media with $10 \%$ Fetal Bovine Serum (FBS). Early experiments showed that HB1.F3 cells expressed Nestin and the ABCG2 transporter, and upon exposure to $5 \%$ bovine serum differentiated into neurons expressing Neurofilament (NF-L, NF-M and NF-H), astrocytes (GFAP) and oligodendrocytes (MBP) ${ }^{51}$. Other human NSC lines used are the ReNCell lines that were derived from 10-week gestation fetal midbrain (ReNCell VM) or 14 week fetal cortex (ReNCell CX), expanded on laminin coated plates in serum free defined NSC media supplemented with EGF, FGF ${ }^{54}$. ReNCells were also immortalized with a retrovirus encoding v-myc (ReNCell VM) or c-myc (ReNCell CX) ${ }^{54}$ and are currently commercially available. ReNCells differentiate upon growth factor withdrawal into early neurons expressing $\beta$ III-Tubulin, dopaminergic neurons expressing tyrosine hydroxylase, GFAP+ astrocytes and oligodendrocytes expressing Galactosylceramide ${ }^{54}$. Despite expression of the myc oncogene, these cell lines have not been shown to produce tumors in the brains of experimental animals or in clinical trials, and most of them do not persist in the brain. The differentiation potential of the HB1.F3.CD line has not recently been tested, nonetheless, it was demonstrated to be safe and effective in trials for glioblastoma.

The main function of NSCs used for the treatment of GBM is to migrate deep into the tumor and deliver therapeutic agents, most often as part of a suicide mission, their ability to differentiate into neurons, oligodendrocytes or astrocytes becoming less relevant. It was demonstrated that NSCs derived from different parts of the mouse and human CNS have different proliferation and differentiation potential and express transcription factors specific for their region of origin ${ }^{32,55,56}$. However, it was also reported that upon culture, expression of many transcription factors that indicate positional identity of the NSCs was downregulated or lost and these changes altered the differentiation potential of these cells ${ }^{57,58}$. It is likely that such changes affect functional aspects of NSCs used for GBM as well; rigorous mechanistic studies in this direction may lead to findings that will improve the efficiency of therapeutic NSCs. 
MSCs are cells capable of differentiating into tissues that are derived from the embryonic mesoderm, such as adipose tissue, cartilage, bone and muscle ${ }^{59}$. MSCs can be found in a great variety of tissues. Therapeutic MSCs have been derived from bone marrow (BM), adipose tissue, umbilical cord blood, and even placenta and have the advantage of being abundant, easy to isolate and propagate, have high tumor-tropic migratory potential and can be donor-matched. Some studies report that unmodified MSCs can promote tumor growth in other cancers ${ }^{60}$. So far, preclinical studies using MSCs for the treatment of glioblastoma have not reported tumor formation (Supplementary Table 1).

NSCs appeared to be better suited in their ability to distribute throughout brain tumors and deliver oncolytic viruses when compared to $\mathrm{MSCs}^{61}$; also, HB1.F3.CD were more efficient in this process than ReNCells ${ }^{62}$. The underlying mechanisms for this therapeutic advantage have so far not been explored. Regrettably, the authors ${ }^{62}$ didn't specify whether the ReNCells used were midbrain or cortex derived (ReNCellVM or ReNCell-CX).

For clinical use and to achieve FDA approval, each newly established SC line must be prepared following Good Manufacturing Practice protocols and requires extensive testing of safety and therapeutic properties according to standards of Good Clinical Practice. When rigorous procedural practices are not followed, as was the case in some for profit clinics that carry out unproven SC interventions for a variety of conditions as well as in the absence of regulatory oversight as encountered in some countries, administration of SCs can lead to major complications, including tumor formation ${ }^{63,64}$, embolism, vision loss, infectious events, autoimmune reactions, stroke, brain hemorrhage and even death ${ }^{14}$.

Induced NSCs (iNSCs), generated by transdifferentiation of somatic cells by transient expression of specific transcription factors, can be derived from the patient's own cells, evading a potential immune response, as may be encountered when using allogeneic NSCs. Both mouse and human iNSCs have been shown effective in preclinical studies for glioblastoma ${ }^{65,66}$. The primary safety concern for therapeutic NSCs is their potential to induce tumor formation, especially when implanted into the brain of patients with established tumors that generate a microenvironment permissive for tumor growth. It was demonstrated in a syngeneic mouse model that iNSCs were safe in this respect, and did not induce tumor formation, unlike induced pluripotent SCs (iPSCs) and embryonic SCs (ESCs) which generated aggressive, deadly tumors ${ }^{67}$. Induced NSCs have not yet been tested in the clinic. Considering the rapid progression of glioblastoma, concerns arise whether such an approach would allow for a timely and in depth quality and safety analyses to validate and expand autologous iNSCs to the necessary amounts needed for therapeutic use, as well as the costs associated with such an endeavor. Nonetheless, transdifferentiation of somatic cells may be a powerful tool for drug delivery. If factors that promote optimal NSC migration and intratumoral distribution are uncovered and engineered in iNSCs, with thorough characterization and testing, the therapeutic NSC toolbox could be considerably expanded. Detailed strategies for generation of iNSCs have been described in a recently published review ${ }^{68}$. 


\section{THERAPEUTIC STRATEGIES AND THEIR MECHANISMS OF ACTION}

The first preclinical studies using SCs for the treatment of malignant glioma emerged twenty years ago, testing four different therapeutic strategies: enzyme/prodrug ${ }^{49}$, oncolytic virus ${ }^{69}$, cytokine therapy ${ }^{70}$ and delivery of pro-apoptotic molecules ${ }^{71}$. Today, these mechanisms are still being tested, optimized, fine-tuned and combined with other treatment modalities to overcome limitations and improve efficacy. Pilot/feasibility and phase I clinical trials using NSCs loaded with oncolytic viruses or delivering enzymes for intratumoral prodrug conversion have recently been completed. Exciting new therapeutic avenues have been opened by the tremendous progress in bioengineering technologies, generating nanoparticles and nanorods that can be conjugated to glioma-tropic SCs for drug delivery and targeted photo-thermal ablation therapy. We describe some of these studies in the following sections, highlighting experimental progress, limitations and efforts to translate these strategies into the clinic.

\subsection{STEM CELLS IN ENZYME/PRODRUG STRATEGIES}

Enzyme/Prodrug strategies for cancer treatment have long been pursued in an effort to artificially create selective and local cytotoxicity for tumor cells, while leaving normal cells unharmed ${ }^{72}$. The most widely used combinations are the Herpes Simplex Virus-Thymidine Kinase (HSV-TK) with Gancyclovir (GCV) and the bacterial Cytosine Deaminase (CD) with 5-Fluorocytosine (5-FC). The HSV-TK enzyme converts GCV into Ganciclovir monophosphate that is further phosphorylated to Gancyclovir triphosphate, a toxic antimetabolite that undergoes erroneous incorporation into DNA, leading to the death of dividing cells (Figure 1A). Cells lacking HSV-TK can still be targeted for apoptosis through a phenomenon called the "bystander effect", which entails transport of the active drug through GAP junctions from neighboring cells, enhancing the cytotoxic response. Similarly, the bacterial enzyme $C D$ is able to convert the nontoxic prodrug 5-FC into the powerful cytotoxic compound 5-fluorouracil (5-FU), that primarily inhibits the production of thymidine, which is required for DNA replication, thus killing dividing cells exposed to it (Figure 1B). Enzyme/Prodrug gene therapy strategies using viral vectors have been extensively explored for the treatment of glioblastoma for over 40 years, but still face many challenges, mainly related to the distribution of the viruses into the tumor ${ }^{73}$. The high migratory capacity of NSCs to distribute throughout the tumor and tumor satellites may serve to improve this therapeutic strategy. 

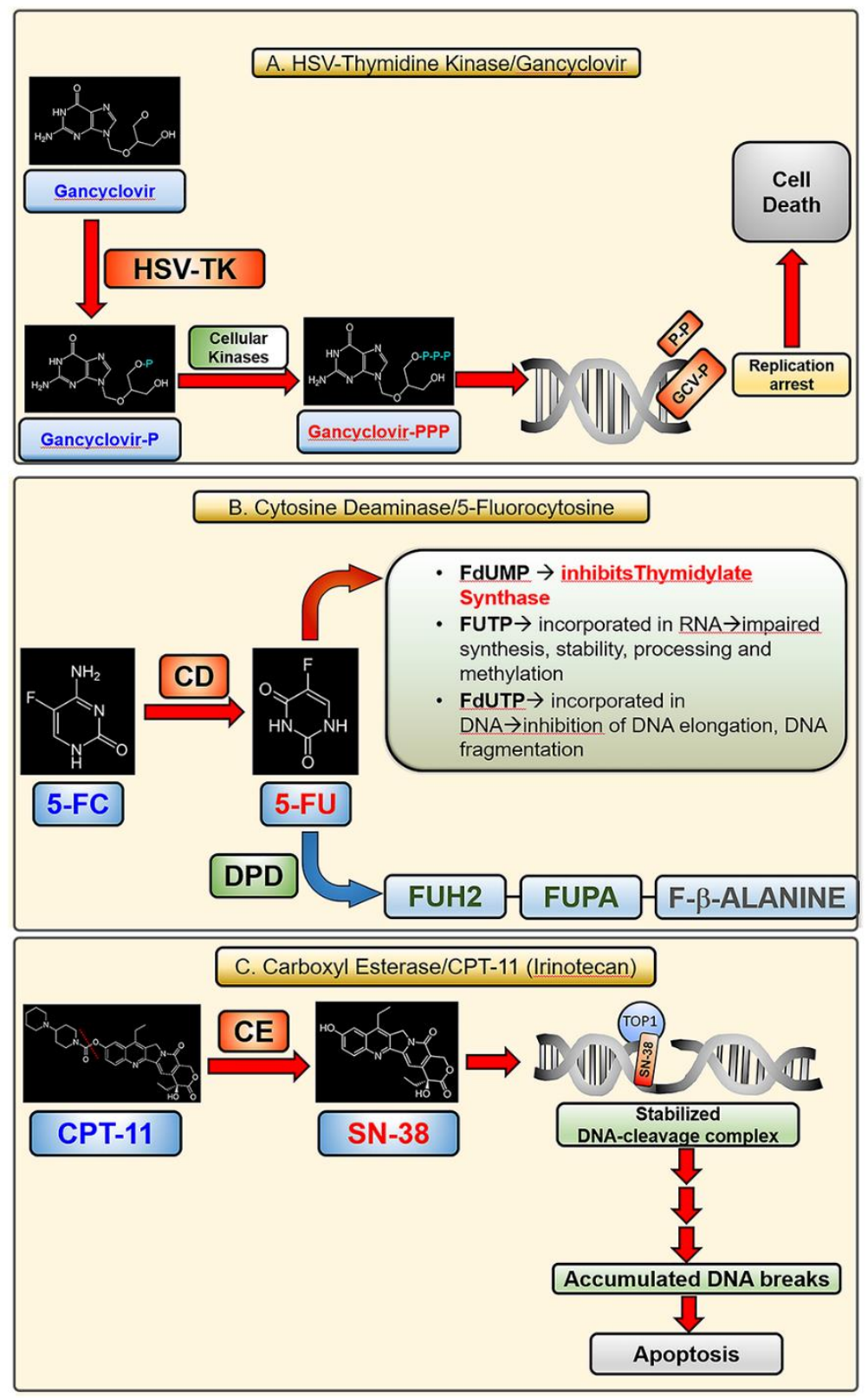

Figure1. Stem Cells in Enzyme/Prodrug Strategies. (A) The Herpes Simplex Virus Thymidine Kinase (HSV-TK) can phosphorylate not only thymidine but also Gancyclovir generating Gancyclovir monophosphate. Cellular kinases convert it into Gancyclovir triphosphate that can be integrated into newly synthesized DNA, arresting further DNA synthesis and inducing cell death. (B) Cytosine deaminase converts 5-Fluorocytosine (5-FC) into 5-Fluorouracyl (5-FU). To exert its cytotoxic function 5-FU is anabolized into fluoro-pyrimidine nucleotides (FdUMP, FUTP and FdUTP). FdUMP (5-Fluoro-2'deoxyuridine 5'-monophosphate) is a suicide inhibitor of the enzyme thymidylate synthase (TS), binding and inactivating it. TS converts dUMP to dTMP. Inhibiting TS results in nucleotide imbalance, excess dUTP and lack of dTMP, leading to DNA damage. FUTP (5-fluorouridine 5'-triphosphate) is extensively incorporated into nuclear and cytoplasmic RNA, leading to impaired RNA synthesis, stability, processing and methylation. FdUTP (5-fluoro-2'-deoxyuridine 5'triphosphate), when incorporated into DNA, inhibits DNA elongation and induces DNA fragmentation. 5FU is deactivated and converted to FUH2 (fluoro-5,6-dihydrouracil) through the catalytic action of dihydropyrimidine dehydrogenase (DPD), the initial and rate-limiting step in the catabolism of 5-FU. FUH2 can be further degraded to FUPA (fluoro- $\beta$ ureidopropionate) and subsequently to FBAL (fluoro- $\beta$-alanine). (C) Carboxyl esterase (CE) converts the water soluble compound CPT-11 into the more potent, lipophilic metabolite SN38. During the DNA synthesis phase of the cell cycle Topoisomerase 1 (TOP1) attaches to the 3 ' end of the cleaved DNA and forms a reversible DNA-TOP1 cleavage complex (TOP1cc). SN-38 binds to TOP1 and stabilizes this complex, halting DNA synthesis and leading to the accumulation of single strand DNA breaks that trigger apoptosis. 
A proof of principle study, using an in vitro co-culture model, demonstrated that NSCs expressing HSV-TK can induce the death of glioma cells when combined with GCV, and this cytotoxic effect was dependent on cell to cell contact and on the presence of the GAP junction protein Connexin $43^{74}$. The bystander effect of HSV-tk delivered by NSCs was also demonstrated in a mouse model in which 66 glioma cells were implanted with NSCs that were transduced with HSV-tk (NSC-tk) ${ }^{75}$. Long-term survival was observed in all animals treated with NSC-tk, whereas all control animals succumbed before 30 days. It was also demonstrated that human BM derived MSCs transduced to express HSV-TK and then injected into mouse brains at the time of glioma cell implantation, induced long term survival in all animals ${ }^{76}$. In a rat glioma model, the same MSC-TK cells improved survival of tumor bearing animals with $40 \%$ of animals becoming tumor-free ${ }^{76}$. These studies demonstrate that delivery of HSV-TK by either NSCs or MSCs provide a significant survival benefit in animal models of glioblastoma. One caveat associated with such studies comes from the simultaneous administration of tumor cells and therapeutic SCs, scenario that cannot be applied in the clinic. Other animal studies have administered SCs at later time following tumor cell implantation, and demonstrated therapeutic effect, albeit with lower efficacy (Supplementary Table 1).

A pioneering preclinical study that illustrated the migration of mouse and human NSCs towards intracranial gliomas ${ }^{49}$, tested the ability of immortalized mouse NSCs $(\mathrm{C} 17.2)^{50}$ that were transduced with cytosine deaminase (CD-NSC) to induce glioma cell death in the presence of 5-FC. Results demonstrated the feasibility of this approach and tumor reduction upon intratumoral administration of CD-NSCs; proliferating NSCs were also killed by the treatment ${ }^{49}$. Human NSC cells HB1.F3 were also transduced to express CD, generating HB1.F3-CD cells that were tested for their migration potential and tumor killing efficacy in mice injected with Daoy medulloblastoma cells ${ }^{77}$. Survival was not analyzed in this study, however HB1.F3-CD cells injected into established tumors, when combined with 5-FC, were able to significantly reduce the tumor volume (by $74 \%$ ) after three weeks of treatment, demonstrating a strong bystander effect from the CD producing NSCs ${ }^{77}$. Adipose tissue derived MSCs (AT-MSC) were transduced with yeast derived Cytosine Deaminase (CDy) and uracil phosphoribosyltransferase (UPRT) generating CDy-AT-MSCs, that migrated towards gliomas when implanted at a distance ${ }^{78}$. UPRT expression catalyzes the conversion of 5-FU to 5-fluorouridine monophosphate, which is more cytotoxic than 5-FU. It was demonstrated that CDy-AT-MSCs improved survival of tumor bearing rats in a dose dependent manner, showing an almost linear dependence of survival to the number of MSCs administered. It was further illustrated that repeated administration of increased numbers of CDy-AT-MSC combined with continuous intraventricular delivery of 5-FC via osmotic pump, further improved survival, with $88 \%$ of animals becoming tumor-free ${ }^{78}$.

Inactivation of $5-\mathrm{FU}$ is dependent on the activity of the rate limiting enzyme Dihydropyrimidine dehydrogenase (DPD) that converts 5-FU to fluoro-5,6-dihydrouracil (FUH2), which is further metabolized to fluoro- $\beta$-alanine (FBAL) (Figure 1B). Several studies have identified an inverse correlation between the sensitivity to 5-FU treatment and levels of DPD, as well as increased toxicity of 5-FU in patients with decreased levels of DPD, oftentimes due to inactivating mutations of $D P D^{79}$. It was also demonstrated that high levels of 
DPD expression in glioma cells can render therapies with hCD-MSC/5-FC inefficient, warranting assessment of DPD levels prior to the start of treatment ${ }^{80}$. Pharmacologic Inhibitors of DPD may also improve efficacy of 5-FU based therapies, especially in local administrations with low systemic load.

The transition from preclinical findings to clinical testing first requires rigorous, diligent, in depth safety analyses. The 2013 study by Aboody and collaborators ${ }^{53}$ thoroughly characterizes the properties of the human NSC line HB1.F3.CD, cell line that was derived from HB1.F3 cells ${ }^{81}$, modified to produce cytosine deaminase. HB1.F3.CD cells were FDA approved as an Investigational New Drug (IND) for therapeutic use in human clinical trials ${ }^{53}$ and to this day, is the only human NSC line used in clinical trials for glioblastoma. It was demonstrated that over 32 passages, HB1.F3.CD cells had a stable, normal karyotype, included just one copy of each of the v-myc and CD genes, retained tropism towards glioma cell lines, even in the presence of dexamethasone and expressed the CD protein and HLA-I antigens but not (or very low levels of) HLA-II. When injected into the brains of naïve immunocompromised or immunocompetent mice (up to $1 \times 10^{\wedge} 6$ cells), no tumor formation was observed; no human cells were detected in the brain or other organs at 4 and 12 weeks after injection, indicating that the HB1.F3.CD cells either did not survive, were cleared by immune cells or both. When administered to mice with intracranial tumors and combined with 5-FC, tumor volume was significantly decreased in animals receiving the highest dose of NSCs $\left(1 \times 10^{\wedge} 5\right)^{53}$. This study highlights the fact that therapeutically efficacy is intimately dependent on the number of NSCs available in the tumor. The pilot/feasibility clinical study designed to use these cells in patients with recurrent high grade glioma (rHGG) (NCT01172964), planned a classic $3+3$ dose escalation regimen ( $1 \times 10^{\wedge} 7$ cells to $5 \times 10^{\wedge} 7$ and 5 -FC from 75 to $150 \mathrm{mg} / \mathrm{kg} / \mathrm{day}$, administered for 7 days, starting 4 days after surgery) with injection of NSCs into the walls of the resection cavity. Results from 15 patients found steady state levels of 5-FU in the brain, much higher than in the blood, indicating local production of 5-FU by the NSCs ${ }^{82}$. Overall, administration of HB1.F3.CD was safe, resulted in no detection of NSCs outside the brain and elicited no humoral immune responses. The median overall survival (OS) at the highest dose level was 15.4 months compared to patients on doses 1-2, with OS of 2.9 months $^{83}$. Distribution of NSCs was analyzed in postmortem brains by v-myc PCR. Several selected sites from brain sections from a male patient, who had died at some time after surgery, tested positive for v-myc by PCR, indicating presence of HB1.F3.CD cells. In situ hybridization with an XY probe (the NSCs are female) on adjacent sections confirmed the presence of the injected NSCs and showed that they were not proliferating. It was estimated that the NSCs traveled about $11 \mathrm{~cm}$ from the site of injection ${ }^{83}$. These results are indeed exciting, proof of safety and of principle, with added optimism for efficacy and identification of live, possibly still therapeutic cells, more than 2 months after administration. The follow up phase I study (NCT02015819) started in 2014, designed to include 18 patients with rHGG, has been modified to include an intraventricular Rickham catheter, placed at the time of surgery, to be used for the administration of subsequent doses of NSCs every two weeks, followed each time by a 7-day course of oral 5-FC. Leucovorin was also added to the protocol to enhance the cytotoxic effects of 5-FU. This study is still ongoing. 
Another enzyme/prodrug combination used for cancer treatment is Carboxyl Esterase (CE) and CPT-11 (Irinotecan), a water soluble semisynthetic derivative of Camptothecin. CPT-11 has antitumor activity on its own, however its lipophilic metabolite SN-38 is a more potent cytotoxic agent. Camptothecin and its derivatives induce DNA damage during the S-phase of the cell cycle by inhibiting Topoisomerase I (TOP-1), forming a stable ternary DNA-TOP1 cleavage complex (TOP1cc) and inducing apoptosis. CPT-11 is metabolized to SN38 by CE, an enzyme naturally present in the liver, albeit at low concentrations (Figure 1C). High expression of $\mathrm{CE}$ in the tumor results in efficient conversion of CPT-11 into SN-38 and high cytotoxic effect, mitigating systemic toxicity. Human Adipose Tissue derived MSCs transduced to express the rabbit CE (hAT-MSC.rCE) were tested in a rat brainstem glioblastoma model, in a protocol that entailed two injections of hAT-MSC.rCE at 2 week intervals with systemic administration of CPT- 11 for 3 weeks ${ }^{84}$. Survival analysis showed a modest improvement (24 days vs. 19 days) in animals treated with hAT-MSC.rCE, highlighting the difficulty of treatment for brainstem gliomas. Administration of CE/CPT-11 was also analyzed in a combinatorial strategy using Amniotic Fluid derived MSCs (AF-MSCs), transduced to express the anti-angiogenic molecule endostatin, and a secreted form of CE. AF-MSCs were injected either intracranially, together with glioma cells or subcutaneously after surgical removal of $90 \%$ of established tumors. AF-MSCs transduced with both CE and endostatin inhibited progression of brain and flank tumors more strongly than AF-MSC expressing only endostatin or SCE and induced decreased vessel density in the tumor, lower proliferation index and increased apoptosis; unfortunately no survival data was presented ${ }^{85}$. A mouse model of medulloblastoma (MB), in which tumors were induced in neonatal mice with intra-cerebellar administration of viruses encoding sonic hedgehog (Shh) and Mycn, genes known to induce MB, was used to test the antitumor effect of NSC-CE/CPT-11 therapy. Human HB1.F3.CD were transduced to express the rabbit CE, that is more effective in metabolizing CPT-11 to SN-38 than the human CE, generating HB1.F3.CD.rCE cells. Animals with established tumors, documented by MRI, were injected into the cerebellum with HB1.F3.CD.rCE then treated systemically with CPT-11. A second dose of NSCs was administered a week later. This regimen resulted in decreased rate of tumor growth, as calculated from MRI data, and a decrease in tumor size when compared to controls at the end of the experiment (postnatal day 71$)^{86}$. Although survival analysis would be difficult in such a model, due to the variability in viral transduction and tumor formation, the study illustrates that NSC mediated CE/CPT-11 enzyme/prodrug therapy for MB is feasible and warrants further optimization.

Administration of the rabbit CE in humans may result in an inflammatory response, reducing the efficacy of the SC treatment. To generate a potent human isoform of CE, Wierdl and collaborators analyzed the structural properties of the rabbit CE enzyme responsible for its increased enzymatic activity and constructed a genetically enhanced version of the human liver CE1. They generated hCE1m6, an enzyme 70-fold more efficient in converting CPT-11 to SN18 than the original CE1 ${ }^{87}$. The HB1.F3.CD human NSC line was transduced with replication deficient adenovirus to transiently produce hCE1m6, generating HB1.F3.CD.hCE1m6 cells. These were compared with HB1.F3.CD.rCE cells in vitro as well as in vivo, using Es1e/SCID mice that have plasma CE levels comparable to those in humans. It was demonstrated that 
HB1.F3.CD.hCE1m6 cells were as efficient as HB1.F3.CD.rCE in converting CPT-11 and had similar migratory behavior and cytotoxic activity against glioma cells. In vitro degranulation assays with the two enzymes using human peripheral blood mononuclear cells, indicated that hCE1m6 had lower immunogenic potential than the rabbit counterpart ${ }^{88}$.

The distribution of HB1.F3.CD.hCE1m6 cells in intracranial glioma tumors was also analyzed following administration into the lateral ventricles, demonstrating that these cells migrated efficiently towards tumors, even in the case of multifocal tumors, located in different hemispheres ${ }^{89}$. NSCs tended to locate around the tumor margin but were also found within the tumor core; some cells aggregated in the ventricles, and this was thought to be due to the speed of the injection or to a cell suspension that was too concentrated (up to $4 \times 10^{\wedge} 5$ cells in 2 ul). It has become evident that optimizing delivery of therapeutic NSCs for maximal tumor coverage necessitates very careful attention to all procedural details. An important step was made with the large scale quantitative analysis by Barish and collaborators ${ }^{90}$ who analyzed the distribution of HB1.F3.CD.hCE1m6 cells, injected either intracranially (i.c.) or intravenously (i.v) into Es1e/SCID mice with orthotopic gliomas. Tumor volumes were calculated from analysis of serial brain sections, number and location of migrating NSCs were scored and the area of the tumor covered was calculated based on the CE molecular weight and diffusion parameters for a tissue like the brain. The careful analysis established, as observed before, that higher doses of NSCs yielded better tumor coverage, but up to a point, when the percentage of actual NSCs present in the tumors declined both in the i.c. and i.v. administration routes. The authors theorize that the rate limiting factors could be tumor dependent or related to the administration technique; high density of cells may lead to aggregation or decreased survival that would limit intratumoral migration and distribution. It was also found that larger tumors attracted more cells, due likely to chemotactic tumor-derived cytokines. Systemic administration required about 10 times more NSCs than i.c injections to reach the same degree of tumor coverage; other parameters of distribution were similar between the two administration routes.

Following in depth analysis of safety, efficacy and pharmacokinetics of the HB1.F3.CD.hCE1m6 cells in combination with i.v. administration of Irinotecan in a mouse glioma model, these cells, identified now as: hCE1m6-NSCs, were approved as an investigational new drug to be tested in a phase I clinical trial for recurrent HGG therapy (NCT02192359) ${ }^{91}$. The study plans to administer hCE1m6-NSCs twice, at two week intervals, and to repeat the cycle every month in the absence of disease progression or toxicities. The goal is to determine the recommended phase II dose of hCE1m6-NSCs, monitor for adverse events, measure SN-38 in the brain and blood, test for immune responses elicited by the allogeneic NSCs and assess potential clinical benefit.

\subsection{STEM CELLS CARRYING PRO-APOPTOTIC MOLECULES}

Dysregulated apoptotic pathways are a hallmark of malignancy. The TP53 tumor suppressor gene, that initiates intrinsic apoptotic pathways in response to cellular stressors, including genomic aberrations and DNA damage is often inactivated in cancer ${ }^{92}$ and very frequently in glioblastoma ${ }^{93}$. Malignant cells evade apoptotic 
signals and therapeutically induced DNA damage and continue to proliferate. Activation of the extrinsic apoptotic pathway via ligands, like the TNF related apoptosis-inducing ligand (TRAIL) or FasL, that bind to cell surface death receptors (DRs) can induce activation of the caspase enzymatic cascade leading to apoptosis independent of TP53. TRAIL has therefore been extensively explored as a promising anticancer agent ${ }^{94,95}$, as it specifically targets tumor cells, leaving normal cells unharmed ${ }^{96}$. Soluble TRAIL is very rapidly cleared from systemic circulation, and strategies for extending its stability and delivery by linking it to nanoparticles (NP) or SC carriers have been intensely studied ${ }^{97}$.

If cells express the TRAIL ligand on their surface they can induce apoptosis in neighboring cells. It was demonstrated that antigen specific CD4 T cells expressing TRAIL can kill glioma cells in vitro ${ }^{98}$. Primary mouse NSCs derived from the forebrain of embryonic mice modified to express human TRAIL were able to specifically induce apoptosis in human glioblastoma cells, in mouse orthotopic xenografts, while sparing the NSC population ${ }^{71}$. Also, mouse NPCs (C17.2), transduced to express a secreted form of TRAIL (S-TRAIL) were able to decrease viability of human glioma cell lines through caspase mediated apoptosis ${ }^{99}$. This effect was synergistically increased if NPC-S-TRAIL was combined with miR-21 knock-down. It had been previously reported that miR-21 is highly expressed in human glioblastoma, and its knockdown led to apoptosis of glioblastoma cell lines ${ }^{100}$. A synergistic effect was found when combining NPCs (C17.2) with Temozolomide treatment. Temozolomide sensitized cells to TRAIL mediated apoptosis by increasing death receptor expression, activating the checkpoint kinase Chk1 and arresting cells in $\mathrm{G} 2 / \mathrm{M}^{101}$.

While many glioma cells are sensitive to TRAIL mediated apoptosis, some, especially GSCs are not. Resistance to TRAIL has been linked to several mechanisms, including upregulation of anti-apoptotic proteins of the Bcl-2 family, inactivating mutations or epigenetic silencing of Caspase 8, or upregulation of FLIP, a molecule that blocks the formation of the death inducing signaling complex (DISC) ${ }^{94}$. In some cells, resistance to TRAIL-induced apoptosis can be overcome by simultaneous administration of the proteasome inhibitor Bortezomib, which results in enhanced Caspase 8 activation that depends on accumulation of the cyclin dependent kinase inhibitor p2 $1^{\mathrm{CIP} 1}$ and inhibition of cyclin-dependent kinase activity (cdk1/2) ${ }^{102}$. In vitro analysis of multiple primary astrocytoma cell lines treated with TRAIL and Bortezomib demonstrated that Bortezomib sensitized these cells to TRAIL-induced apoptosis by increasing the expression of the signaling death receptors TRAIL-R1, or TRAIL-R2, enhancing formation of the DISC complex, increasing recruitment of Caspase 8 to DISC and decreasing recruitment of the inhibitory protein FLIP ${ }^{103}$ (Figure 2). Balyasnikova and collaborators tested the combinatorial use of TRAIL, delivered by NSCs, with Bortezomib in a preclinical orthotopic glioma model ${ }^{104}$. This study demonstrated that NSC-TRAIL, when injected at a 1:1 ratio with tumor cells at the time of tumor initiation, was able to induce long term survival in $80 \%$ of mice. This was increased to $100 \%$ long term survival when Bortezomib was added. The result was dose and time dependent; lowering the number of NSCs or administration of NSCs into established tumors decreased the number of long term survivors. Administration of Bortezomib increased expression of the TRAIL-R2 receptor in vitro, that could 
account for the increased sensitivity to TRAIL. This effect was not reproduced in vivo, possibly due to poor penetration of the drug into the tumor, following systemic administration ${ }^{104}$.

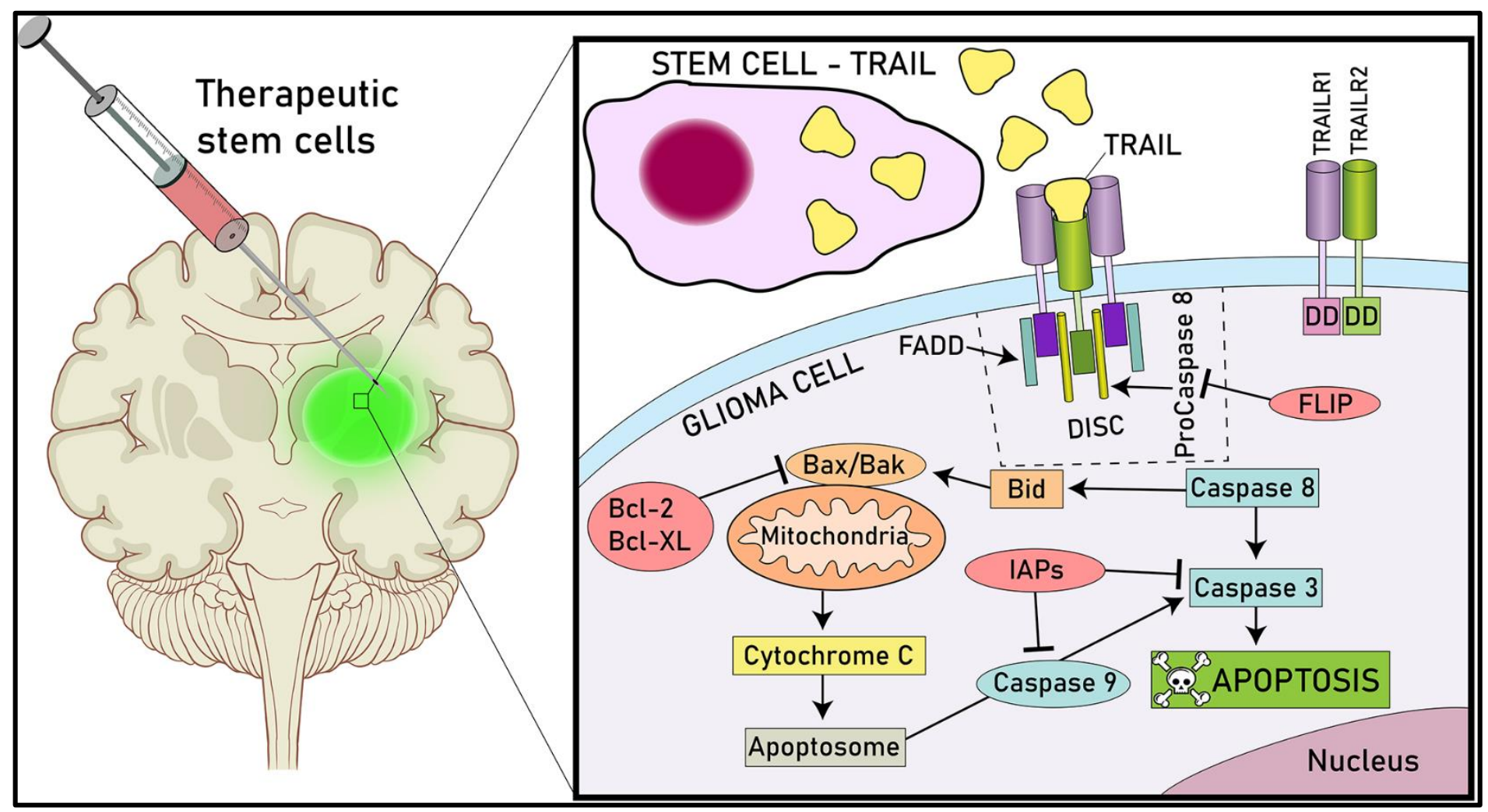

Figure 2. Stem Cells carrying pro-apoptotic molecules. Intratumoral delivery of the TNF related apoptosis-inducing ligand (TRAIL) activates of the extrinsic apoptotic pathway. Binding of TRAIL to the death receptors DR4 (TRAIL-R1) and DR5 (TRAIL-R2) results in formation of the cell Death Inducing Signaling Complex (DISC) and proteolytic cleavage of Procaspase 8. This triggers activation of the effector Caspase 3 leading to apoptosis. Activation of the initiator Caspase 8 results also in release of inhibition of the proapoptotic molecules Bax and Bak, release of Cytochrome $\mathrm{C}$ from the mitochondria and further activation of the effector caspases. Resistance to TRAIL induced apoptosis may be caused by excess FLIP. FLIP binds to FADD and Caspase-8 and inhibits formation of the DISC complex and activation of Caspase 8. The proteasome inhibitor Bortezomib increases sensitivity of tumor cells to TRAIL induced apoptosis by inhibiting recruitment of FLIP to the DISC complex and increasing expression of the two signaling TRAIL receptors (TRAIL-R1 and TRAIL-R2).

TRAIL was also delivered into the brain of tumor bearing rats via iron oxide magnetic nanoparticles (NPs), and it was shown that NP-TRAIL were effective in inducing apoptosis of glioma cells and significantly extended the MS of tumor bearing rats when compared to rats treated with free soluble TRAIL ${ }^{105}$. NP-TRAIL were also able to induce apoptosis and increase radiation sensitivity and efficacy of the proteasome inhibitor Bortezomib in patient derived Glioma Stem Cells (HF2414, HF2584), cells refractory to TRAIL induced apoptosis ${ }^{105}$.

In addition to established allogeneic Neural Stem and Progenitor Cell lines that may be in limited supply and may induce immunological rejection, other SCs have been explored as cell carriers for TRAIL, providing an avenue for autologous cell therapies for glioblastoma treatment. Human adipose derived MSCs transduced with TRAIL using biodegradable NPs were able to migrate towards intracranial tumors and selectively induced 
glioma cell death via caspase mediated apoptosis, while sparing normal astrocytes. Treatment with hADMSCTRAIL significantly extended the MS of glioma bearing animals, especially after repeated intratumoral administrations ${ }^{106}$.

TRAIL mediated apoptosis of glioma cells has also been accomplished using iNSCs. Mouse Embryonic Fibroblasts (MEFs) were transdifferentiated with lentiviral expression constructs of Brn2, Sox2 and FoxG1 to generate iNSCs. Glioma tropism of these cells was demonstrated in vitro and in vivo and when transduced to express sTRAIL ${ }^{65}$. Administration of iNSC-sTRAIL 3 days after implantation of patient derived cells (GBM8) extended the survival of tumor bearing animals from 37 to 59 days ${ }^{65}$. A subsequent study from the same group $^{66}$ reported generation of human iNSCs ( $\mathrm{h}-\mathrm{iNSC}^{\mathrm{TE}}$ ) by transdifferentiating and immortalizing human fibroblasts with lentiviral vectors encoding SOX2 and human telomerase (h-TERT). Transcriptionally, these cells resembled brain derived NSCs and migrated selectively towards glioblastoma cells and spheroids in vitro, in a CXCR4 dependent manner. When injected into the brain of immunodeficient mice iNSCs persisted for up to three weeks, but were eventually cleared. These cells were then transduced to express either a secreted form of TRAIL (h-iNSC ${ }^{\text {TE }}$-TRAIL) or Thymidine Kinase (TK): h-iNSC ${ }^{T E}-T_{K}$ and tested as therapeutic agents. The h-iNSC ${ }^{T E}$-TRAIL reduced viability of glioma cells in vitro and extended the MS of mice bearing intracranial xenografts when equal numbers of tumor cells and h-iNSC ${ }^{\mathrm{TE}}$-TRAIL were implanted at the same time. Intratumoral administration of $\mathrm{h}$-iNSC ${ }^{\mathrm{TE}}-\mathrm{TK}$ when combined with GCV also increased MS of glioma bearing mice. The group further went on to demonstrate that post-surgical treatment with h-iNSC ${ }^{\mathrm{TE}}$-TK cells, encapsulated in ECM hydrogel that was placed into the tumor cavity, followed by GCV administration, a paradigm that more closely resembles the clinic, also resulted in an increased MS of animals ${ }^{66}$.

An interesting development of cell mediated therapy for glioblastoma is represented by the use of modified tumor cells to deliver therapeutic agents into the tumor. This is based from observations of the "selfseeding" behavior of cancer cells, where metastatic cells migrate back toward the primary tumor or cells from the primary tumor migrate toward established metastase ${ }^{107}$. Glioma cells, especially GSCs, share many characteristics with NSCs, including migration and invasion towards CXCL12 and TGFb ${ }^{108,109}$. Genetically modified glioma cells have therefore been tested for their ability to deliver pro-apoptotic molecules for the treatment of glioblastoma and other metastatic cancers ${ }^{110}$. Two strategies for this approach were designed: allogeneic "off the shelf" glioblastoma cells, intrinsically resistant to TRAIL mediated apoptosis and expressing a secreted form of TRAIL (sTRAIL), or autologous glioma cells with CRISPR/Cas9 mediated genetic deletion of the two TRAIL signaling receptors (TRAIL-R1(DR4) and TRAIL-R2(DR5)) and lentiviral expression of sTRAIL. The cells were also modified to express HSV-TK, to render them sensitive to GCV mediated elimination, yet insensitive to the actions of TRAIL. Mice with established intracranial tumors injected with these cells showed increased median survival, efficiency being further enhanced with GCV administration ${ }^{110}$.

Overall, these studies demonstrate that SC represent efficient vehicles for delivery of TRAIL into the tumors and that the therapeutic effect is dependent on the tumor sensitivity to TRAIL mediated apoptosis. This 
strategy may be useful in combinatorial approaches with agents that mitigate resistance to TRAIL and other synergistic cytotoxic drugs.

\subsection{STEM CELLS AND ONCOLYTIC VIROTHERAPY}

Oncolytic viral therapy uses viral vectors that are either able to selectively replicate in tumor cells and induce tumor cell lysis that is propagated outwards from the site of virus administration, while sparing normal brain cells, or employs viruses that are replication-deficient and are used for the delivery of therapeutic genes $^{111,112}$. In addition to the direct tumor lytic effect, oncolytic viruses strongly stimulate the innate immune response through viral antigens (PAMPS: Pathogen Associated Molecular Patterns) and the anti-tumor immune response through the release of Damage-Associated Molecular Patterns (DAMPs), tumor associated antigens (TAA) and neoantigens, inducing immunogenic cell death (ICD) ${ }^{113}$ (Figure 3). The idea to use viruses to treat malignancies emerged from observations that concomitant viral infections, like influenza, had beneficial effects on the progression of blood malignancies. For almost a century, numerous viruses have been modified and optimized for use in the treatment of cancer ${ }^{114}$. Today, exquisitely tailored adenoviruses, HSV, vaccinia, measles, retro- and reoviruses are being tested in clinical trials as emerging anti-glioma therapies that bring hope for a cure ${ }^{115}$. The most compelling of these: PVS-RIPO, TOCA511, and DNX-2401 have been granted an expedited drug review process by the FDA ${ }^{115}$. The PVS-RIPO, a recombinant nonpathogenic poliovirus that is recognized by the poliovirus receptor CD155, present on many tumor cells, has been proven safe in a Phase I trial with WHO grade IV malignant glioma (NCT01491893), with patients showing an increased survival rate compared to historical controls ${ }^{116}$. Repeated administration of the virus in patients in which the disease recurred was also concluded to be safe, with encouraging efficacy results ${ }^{117}$. The TOCA511 virus is a replicating gamma retrovirus, based on a modified murine leukemia virus, that also encodes cytosine deaminase (CD). In the TOCA511 ascending phase I trial (NCT01470794), 56 patients with rHGG were treated with intra-cavity administration of TOCA511 and orally administered 5-FC; durable complete responses were observed, with the trial entering phase III $^{118}$. DNX-2401 (Delta-24-RGD), a replication-competent oncolytic adenovirus has been tested in a phase I trial in 37 patients with rHGG; evidence of long term survival, direct oncolytic effects and immunological antitumor effects were observed ${ }^{119}$. In phase III, the TOCA511 trial (NCT02414165) was tested in 403 patients with rHGG or anaplastic astrocytoma (AA) and compared to standard of care (SOC). Results indicated compelling improvement of overall survival (OS) in patients with secondary recurrence, and favorable trends in IDH1 mutant and AA population. Overall, there were no significant differences in OS between the two arms ${ }^{120}$. Exciting results have also been reported from Japan with the third-generation oncolytic Herpes Virus $\mathrm{G} 47 \Delta$, that has been engineered for enhanced viral growth and MHC-I presentation in infected tumor cells ${ }^{121}$. A phase II trial with this virus in 13 patients with recurrent or residual glioblastoma revealed an increased survival rate in virus treated patients compared to historical controls and also increased antitumor immune responses with elevated infiltration of $T$ cells at the injection sites $^{122}$. 


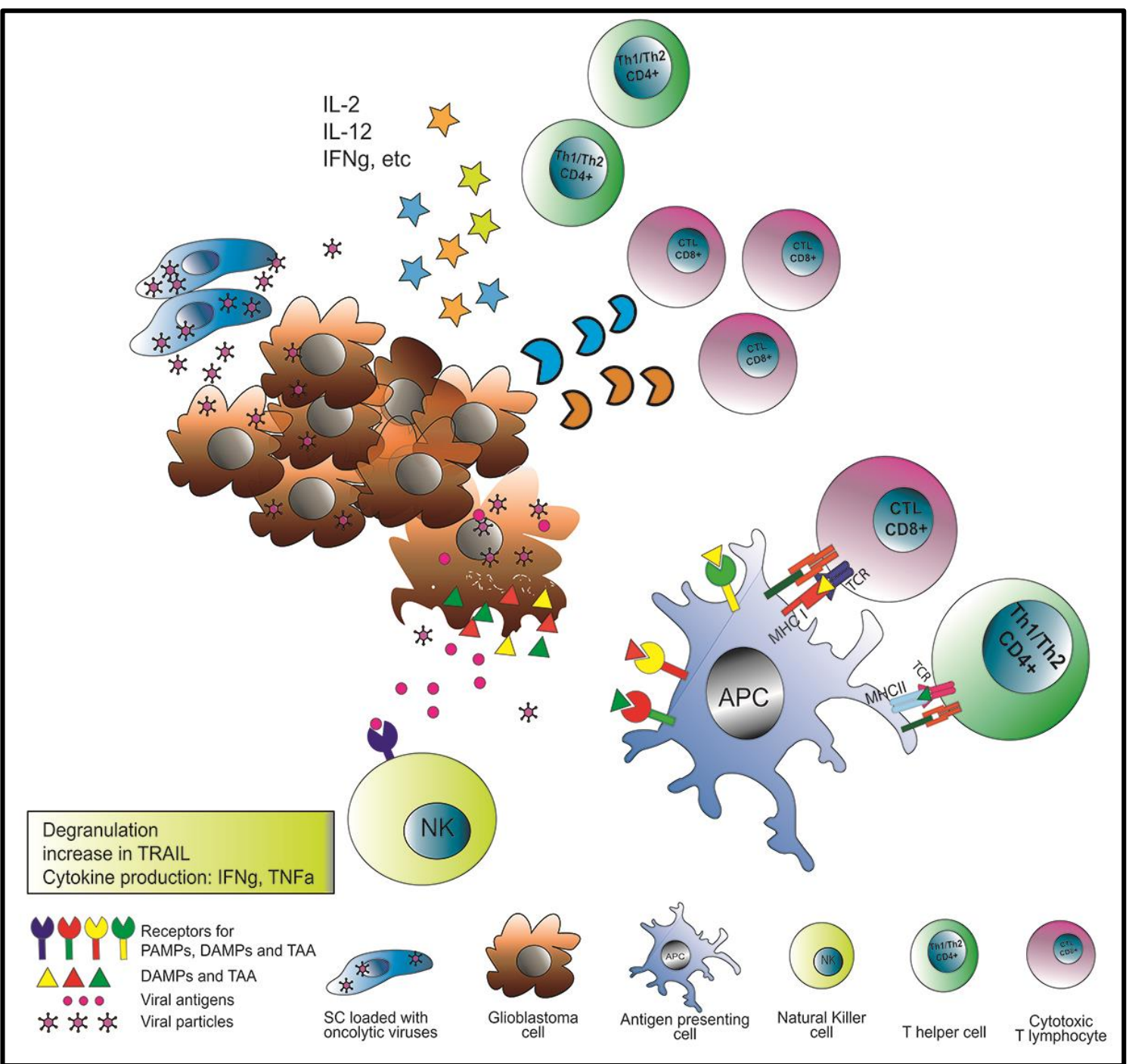

Figure 3. Stem Cells and Oncolytic Virotherapy. Intratumoral delivery of oncolytic viruses by Stem Cells results in virus induced glioma tumor cell death and also in immunogenic cell death (ICD) through activation of the innate and adaptive antitumor immune responses. Dying cells release viral antigens (Pathogen Associated Molecular Patterns, PAMPs), Damage Associated Molecular Patterns (DAMPs) and Tumor Associated Antigens (TAA). These antigens activate NK cells that induce direct tumor cell killing through production of granzyme and perforin, and also induce apoptosis through the release of TRAIL, TNF $\alpha$ and IFN $\gamma$. DAMPs and TAA activate Antigen Presenting Cells that travel to the draining lymph nodes where they cross present antigens to naïve $T$ lymphocytes and activate them. Activated $T$ helper and Cytotoxic $T$ lymphocytes migrate into the tumor and release cytokines, perforin and granzyme that amplify the cytotoxic effect.

Taken together, these clinical studies are indeed exciting, and provide hope that oncolytic virotherapy may provide therapeutic benefit. Nonetheless, limitations exist, particularly in relation to the limited spatial distribution of the virus following injection, especially in large tumors and those that invade at a distance and necessitate multiple rounds of injections at different coordinates. As a result, many oncolytic viruses (OVs) designed to specifically replicate in glioma cells that were shown to be safe in early clinical trials did not 
advance to phase III studies ${ }^{123}$. Tumor tropic SCs may be able to improve viral distribution and enhance therapeutic efficacy, especially in tumors that are difficult to reach surgically.

Numerous preclinical studies have demonstrated an advantage of using SCs as vehicles for the delivery of OVs in intracranial gliomas. Several strategies have been employed to allow specific replication of Conditionally Replicating Adenoviruses (CRAds) in glioma cells: deleting a viral region responsible for inactivating $\mathrm{p53}$, as in the ONYX-015 virus ${ }^{124}$, or deleting the $\mathrm{E} 1 \mathrm{~A}$ viral region that binds to Retinoblastoma $(\mathrm{Rb})$, allowing for replication in cells with defects in the Rb pathway, frequently altered in glioblastoma, as in the Delta24 virus ${ }^{125}$. Later it was established that the ONYX-015 was also active in cells with intact p53, and the selectivity for tumor cells was dependent on a tumor specific viral RNA export mechanism ${ }^{126}$. The Delta24 virus was further modified to express the RGD sequence (Arginine-Glycine-Aspartic Acid), improving infectivity of tumor cells, and evolved into the very promising DNX2401/DELTA-24-RGD. Further modifications of DNX2401 resulted in $3^{\text {rd }}$ generation CRAd vectors, like the ICOVIR15, which encodes an enhanced E1A promoter for optimal viral function while maintaining a small genome size ${ }^{127}$. The ICOVIR17 was generated from ICOVIR15, modified to express hyaluronidase for enhanced intratumoral distribution of the virus ${ }^{128}$. ICOVIR17 was further enhanced with an RGD insertion on the fiber shaft, rather than the fiber knob, for increased tumor cell infectivity generating VCN-01 ${ }^{129,130}$. Administration of MSCs loaded with ICOVIR17 significantly enhanced MS in tumor bearing animals when compared to virus administration alone; the presence of the hyaluronidase in ICOVIR17 also conferred increased survival advantage over ICOVIR15. Encapsulating ICOVIR17 infected MSCs in a synthetic extracellular matrix (sECM) that was implanted in the post-resection cavity of mice with intracranial tumors also showed higher efficacy compared to administration of virus alone ${ }^{131}$. Similarly, encapsulated MSCs carrying the oncolytic Herpes Virus G47 $\Delta$ killed tumor cells more efficiently than administration of virus alone, and arming G47 $\Delta$ to express TRAIL further enhanced the survival of tumor bearing mice ${ }^{132}$.

Myxomavirus is a virus pathogenic to European rabbits, but not other vertebrates, making it a safe therapeutic oncolytic virus. This virus was used to infect adipose derived MSCs generating hADMSC-vMyxo cells. Treatment of glioma bearing animals with hADMSC-vMyxo induced their long term survival and repeated administrations of MSCs enhanced the therapeutic benefit ${ }^{133}$.

Specific replication of CRAds in glioma cells was also accomplished by employing the human CXCR4 promoter to drive expression of the viral E1 gene (CRAd-CXCR4-RGD, and CRAd-CXCR4-5/3); hMSC infected with these viruses were able to migrate into the tumor and deliver a higher dose of viral particles, especially at distant pockets of migrating glioma cells than free viral particles ${ }^{134}$. The CXCR4 promoter allowed replication of this virus in both hMSCs and tumor cells, but not in normal astrocytes. Toxicity of the virus was significantly lower in hMSCs than in tumor cells ${ }^{134}$.

Survivin, a member of inhibitor of apoptosis (IAP) family is specifically expressed not only in cancer cells and cancer stem cells, but also in normal SCs where it promotes survival and proliferation ${ }^{135}$. Generating CRAds that drive expression of the E1A viral replication gene under the control of the survivin promoter 
allowed for great specificity of the oncolytic effect. CRAd-S-pk7 was engineered with survivin promoter dependent replication and a poly-lysine modification (pk7) that binds to heparin sulfate proteoglycans expressed on the surface of tumor cells, facilitating viral entry. This virus was able to infect both NSCs and human glioma cells with high efficiency, allow NSCs to migrate into orthotopic implanted tumors in mice and reduce the size of glioma flank tumors by about $50 \%$, when compared to administration of loose viral particles $^{136}$. The same CRAd-S-pk7 vector was used to optimize parameters of viral loading of NSCs to achieve relevant therapeutic efficacy that can be explored clinically ${ }^{137}$. The study demonstrated that loading the adenovirus onto NSC carriers at a low dose (10-50 viral particles/cell) allowed virus replication with reduced lysis of NSCs, but efficient killing of glioma cells. This strategy mitigated virus induced neuro-inflammation, enhanced tumor-tropic migration of NSCs and prolonged the MS of tumor bearing mice by about 30\%, when compared to administration of loose viral particles in an orthotopic glioma model ${ }^{137}$. It was then demonstrated that the human NSC immortalized cell line (ReNCell) was more effective in delivering viral particles to glioma cells compared with $\mathrm{hMSCs}^{61}$. The ReNCells released more viral particles than MSCs following viral infection and displayed enhanced migration towards tumor cells. When implanted in mice with U87MG intracranial tumors ReNCells laden with CRAd-S-pk7 were more effective than hMSCs in killing glioma cells, improving the MS of tumor bearing mice ${ }^{61}$. A further study found that the HB1.F3.CD human NSC line displayed increased efficacy compared to ReNCells in delivering the CRAd-S-pk7 to U87MG and improved median survival of tumor bearing animals ${ }^{62}$. It remains unknown why NSCs have higher capacity of oncolytic virus delivery compared to other cells. Until underlying mechanisms are identified, therapeutic SCs will each need to be individually and thoroughly characterized, keeping in mind that even in the most strictly controlled and optimized preclinical studies there is variability from one study to the next.

Analysis of experimental details from successful preclinical trials (Supplementary Table 1) reveals that in order to be effective in inducing tumor cell death, high numbers of SCs (close to the number of tumor cells) need to be effectively present within the tumor. This has been accomplished by delivering a high number of SCs directly into the tumor and by repeated administrations. Local administration into the brain is invasive and repeated administration may lead to complications. An alternative route of non-invasive administration of therapeutic SCs is represented by intranasal delivery, taking advantage of the anatomical and physiological properties of the nasal mucosa, allowing for transport alongside the olfactory or trigeminal nerves, and through the perivascular pathway within the CNS, avoiding transport restrictions imposed by the BBB and clearance of therapeutic cells following systemic administration ${ }^{138}$. Knowing that tropism of SCs towards gliomas is promoted by the CXCL12/CXCR4 signaling axis and that expression of CXCL12 in gliomas is induced by hypoxia and irradiation ${ }^{41,42,139}$, Dey and collaborators tested whether overexpression of CXCR4 on the human NSC line HB1.F3.CD would improve intratumoral oncolytic virus delivery, following repeated intranasal administration of NSCs infected with CRAd-S-pk $7^{140}$. The authors demonstrated that hypoxic preconditioning and transduction of NSCs with CXCR4 promoted migration of NSCs towards glioma cells and increased survival of mice bearing intracranial xenografts treated with radiation therapy (XRT) prior to intranasal delivery 
of HB1.F3.CD infected with CRAd-S-pk $7^{140}$. Interestingly, treatment with XRT and oncolytic virus loaded NSCs in the absence of hypoxia preconditioning or expression of CXCR4 did not improve survival over XRT alone. Previously, it was shown that HB1.F3.CD cells infected with CRAd-S-pk7 were effective in improving survival of mice with GBM43 xenografts when radiation was administered after intracranial delivery of NSCs, and less so when XRT was administered prior to NSCs ${ }^{141}$. These studies illustrate that the timing of NSC administration relative to radiation therapy and the route of administration are critical factors to consider when combining OVNSCs with XRT.

To improve the delivery of SCs using the intranasal (IN) route, Spencer and collaborators tested two methods of reducing the clearance of NSC from the nasal cavity and improving transmigration of NSCs through the nasal epithelium into the brain: administration of a biodegradable fibrin and thrombin based glue into the nasal cavity immediately after the administration of NSCs loaded with OV, or administration of methimazole, a drug used to treat hyperthyroidism, that also causes a thinning of the nasal epithelium ${ }^{142}$. Using the same HB1.F3.CD NSC line, overexpressing CXCR4 and loaded with CRAd-S-pk7, the authors demonstrated that both the fibrin glue and methimazole decreased the clearance of IN administered NSCs and that methimazole treatment increased migration into the tumor and extended the survival of tumor bearing mice treated with OV loaded NSCs. In the absence of methimazole, IN OV-NSCs induced no survival benefit ${ }^{142}$.

In preparation for clinical use, the HB1.F3.CD NSC cell line, loaded with CRAd-S-pk7 (now named: NSC-CRAd-S-pK7) has been extensively tested in laboratory animals for tumor coverage and distribution ${ }^{143,144}$, safety, viral delivery, persistence in immunocompetent semi-permissive hosts ${ }^{145}$ and efficacy when combined with standard of care ${ }^{141}$. It was demonstrated that administration of NSC-CRAd-S-pK7 is safe, results in extensive coverage of the tumor area by migrating NSCs and is more effective when administered prior to radiation and temozolomide therapy. Evidence was presented in support of a mechanism by which pretreatment with CRAd-S-pk7 impaired the DNA damage response and increased radio-sensitivity of glioma cells ${ }^{141}$.

The human HB1.F3.CD loaded with the CRAd-Survivin-pK7 oncolytic virus has been approved by the FDA as a clinical grade agent. The first in human phase I clinical trial for primary grade III and IV gliomas using NSCs as delivery agents for oncolytic viruses (NCT03072134, 2017-2020) was recently completed ${ }^{146}$. The study enrolled 13 patients with resectable or unresectable tumors, with the primary goal of establishing safety and toxicity of the NSC-CRAd-S-pK7 therapy when combined with SOC, including a dose escalation regimen with three doses of NSCs: 50, 100 and 150 million cells, corresponding to $6.25 \times 10^{\wedge} 10,1.25 \times 10^{\wedge} 11$ and $1.875 \times 10^{\wedge} 11$ viral particles respectively, which were injected at the site of tumor biopsy or resection, followed by standard radiotherapy and Temozolomide treatment. The study also aimed to follow tumor progression by MRI and measure changes in cytokines and T cell subgroups ${ }^{146}$. Results from this study, not yet revealed, are expected with heightened anticipation. 


\subsection{THERAPEUTIC CYTOKINES, ANTIBODIES AND TOXINS DELIVERED BY STEM CELLS}

Developing effective immunotherapy strategies for glioblastoma has been the goal of numerous preclinical and clinical studies over many decades, with limited success so far. New enthusiasm followed successes in the treatment of other solid tumors with checkpoint inhibitors and sustained efforts to replicate such results in glioblastoma are ongoing ${ }^{147}$. The promise of long-term efficacy by eliciting durable antitumor immunological memory is a highly desired outcome, especially in a cancer that inevitably recurs.

Immunotherapy for gliomas needs to address the severe tumor-induced immune suppression, in addition to being mindful of the minimal tolerance for inflammation in the brain ${ }^{148}$. In an effort to boost the antitumor immune response and aid antiglioma vaccine therapies, cytokine therapy has been pursued in preclinical and clinical trials for glioblastoma. The most promising results were shown with delivery of IL-2, IL-4, IL-12, and GMCSF ${ }^{149,150}$. In addition to antigen presenting cells (APCs) that bring the added advantage of expressing costimulatory proteins able to directly activate immune cells, glioma tropic stem cells represent good vehicles for cytokine delivery.

IL-4 strongly activates the antitumor immune system in a variety of cancers ${ }^{151,152}$. Expression of the IL-4 receptor has been found extensively on glioblastoma cells, and not on normal neurons and astrocytes, representing a good target for specific therapeutic intervention ${ }^{153}$. The therapeutic potential of mouse and rat NPCs, genetically modified to produce IL-4, has been tested in immunocompetent preclinical models of glioma $^{70}$. Mouse NPCs were generated from the cortex of a postnatal day 1 mouse and transduced with a retrovirus to express IL- $4^{70}$. It was demonstrated that mouse NPC-IL4 induced long term survival in the majority of mice when injected into the brain at the same time with tumor cells and when injected 5 days later into established tumors. Similarly, NPCs, isolated from the striatum of a rat and immortalized with a temperature sensitive variant of the SV-40 Large T antigen, transduced to express IL-4, induced increased survival of animals with new or established tumors when compared to control animals and even when compared to retrovirus-mediated, in vivo transfer of IL-4 vectors ${ }^{70}$.

IL-12, a cytokine with important roles in modulating crosstalk between the native and adaptive immune system, induces proliferation of T lymphocytes and NK cells and stimulates production of other cytokines, especially of IFN $\gamma$. These activities result in a strong antitumor response in numerous cancers ${ }^{154,155}$. It was shown that intracranial administration of mouse NSCs transduced to express IL-12 improved survival in immunocompetent C57BL/6 mice ${ }^{156}$. Importantly, animals that survived the first administration of tumors were able to reject tumors implanted into the contralateral hemisphere three months later, demonstrating development of antitumor immunological memory. Increased infiltration of CD4 and CD8 T cells into the tumor was also observed. ${ }^{156}$. Similarly, in a rat glioma model, intratumoral administration of human NSCs, that were isolated from the hippocampi from 3-5 months old human embryos and transduced to express IL-12, significantly improved median survival (MS) of tumor bearing rats from 17 to 87 when NPC were administered at the same time with tumor cells, or to 73 days when NPCs were administered 5 days later ${ }^{157}$. Increased infiltration with T cells was also observed. In this study, the large survival benefit was likely due to the high 
number of administered hNSCs: 10 and 30-fold higher than the number of tumor cells injected ${ }^{157}$. MSCs derived from either mouse $\mathrm{BM}^{158}$ or human umbilical cord ${ }^{159}$ modified to express IL-12 were also found to provide therapeutic benefit in preclinical glioma models.

Evidence that cytokine delivery by SCs induces immunogenic cell death (ICD) of glioma cells in preclinical models is provided by an elegant study with mouse BM derived NSC-like cells, transduced to express IL-23 (BM-NSC-IL23), a cytokine that induces IL-12 and IFN $\gamma$ production ${ }^{160}$. Sixty percent of mice treated with BM-NSC-IL23 showed long term survival (>60 days) in immune competent B6 mice, while the MS of control animals was about 30-35 days. Antibody depletion of either CD8+ cytotoxic cells (CTLs), CD4+ or NK cells reduced the survival benefit of tumor bearing mice, demonstrating that CTLs were the primary cells responsible for the observed effect with minor contributions from CD4+ and NK cells ${ }^{160}$. When surviving animals were re-challenged with glioma cell implantations, they rejected the tumors, becoming tumor free, indicating establishment of long term antitumor immunity; this process was associated with increased levels of IFN $\gamma$. Studies using MSCs that delivered IL-18, IFN $\beta$ or both cytokines also showed a therapeutic effect and establishment of long-term anti-tumor immunity in glioma bearing rats, an effect associated with increased intratumoral production of IL-2 and IFN $\gamma^{161,162}$. Combined delivery of IL-7 by MSCs with peripheral immunization, with IFN $\gamma$ transduced irradiated tumor cells also resulted in decreased tumor size in a rat model of glioma ${ }^{163}$. MSCs transduced to express IFN $\beta$ had a direct cytotoxic effect on mouse glioma cells and human primary GBM cells and induced survival benefit in tumor bearing mice, an effect that was more pronounced when MSC-IFN $\beta$ was placed in the post-resection cavity and encapsulated in a synthetic ECM ${ }^{164}$. The melanoma differentiation associated gene-7 (mda-7/IL-24) cytokine, expressed in terminally differentiated cells and less so in malignant cells, also induces a direct, specific cytotoxic effect in a variety of tumor cells and increases sensitivity to radiotherapy ${ }^{165}$. It has been modified for increased stability and expression of luciferase, for in vivo luminescence tracking, generating the multifunctional protein SML7 ${ }^{166}$. Primary mouse NSCs, transduced to deliver SML7 induced a reduction in intracranial glioma growth; co-administration of STRAIL by NSCs, further improved this beneficial effect. ${ }^{166}$.

BMP4, a critical developmental growth factor in embryogenesis, was reported to inhibit the tumorigenic potential of brain tumor initiating cells (BTIC) and increase survival of glioma bearing animals when administered into the tumor as BMP4 coated acrylic beads ${ }^{167}$. Treatment with human adipose derived MSCs (hAMSCs), transduced with a retroviral vector to express BMP4 and administered systemically into the left cardiac ventricle of mice with intracranial gliomas (U87 cells), also elicited great survival benefit, with over $75 \%$ of tumor bearing animals becoming tumor free ${ }^{168}$. To increase safety of the therapeutic MSCs, avoiding possible insertional mutagenesis or other viral antigen induced inflammatory-responses, hAMSCs were transfected to express BMP4 using polymer nanoparticles (NP-BMP4.hAMSCs) ${ }^{169}$. Conditioned medium from these cells decreased the viability of patient derived human brain tumor initiating cells (BTIC) in vitro. Intranasal administration of NP-BMP4.hAMCs in rats bearing intracranial tumors with BTIC significantly improved MS from about 14 days to about 17 days. It was also demonstrated using fluorescence imaging that 
both intravenous and intranasal administration of these MSCs resulted in extensive distribution into the tumor $^{169}$. The difference in the extent to which the two BMP4.hAMSCs preparations increased survival in the mouse vs. the rat model is large: $75 \%$ of mice were tumor free vs. a $\sim 3$-day increase in MS in rats. However, the studies cannot be properly compared due to other variations in the two experimental paradigms. The difference could be explained by the different distribution of MSCs following intracardiac vs. intranasal delivery, but is most likely due to the different tumor cells used, highlighting the fact that impressive results when using the U87 glioblastoma cell line may not reflect efficacy on primary patient tumor BTICs.

Another strategy for cancer immunotherapy, kindled by advances in toxinology and immunology, emerged in the late 1970s, when hybrid molecules were constructed from potent toxins conjugated to antitumor antibodies to specifically target tumor cells, leaving normal cells unharmed ${ }^{170}$. Debinski and collaborators demonstrated that the receptor for IL-13 is amply expressed on numerous glioblastoma cell lines, and that a chimeric protein made of the human IL-13 and a modified form of Pseudomonas exotoxin (PE): PE38QQR had a powerful cytotoxic effect on glioblastoma cells that was neutralized by IL-13 ${ }^{171}$. It was later discovered that IL-13 binds with high affinity to IL13R $\alpha 2$, which is present almost exclusively on tumor cells, acts as a decoy receptor and has a higher affinity for IL-13 than IL13R $\alpha 1$, a receptor found on most normal cells ${ }^{172}$. IL13-PE38QQR was embraced with great enthusiasm and developed as an investigational drug. A phase III multicenter randomized controlled study in first recurrence glioblastoma patients comparing BCNU wafers with IL13-PE38QQR administered by convection enhanced delivery through a catheter placed into the resection cavity, found no significant difference in OS between the two groups ${ }^{173}$. The authors had concerns about the distribution of the drug, especially to tumor cells that invaded away from the resection cavity, the short half-life of the chimeric protein and the heterogeneous intratumoral expression of IL13Ra2. To overcome these limitations, a stem cell based distribution strategy was designed. Since PE acts by inactivating the elongation factor EF-2 which leads to inhibition of protein synthesis, EF-2 can be modified to prevent the toxin from exerting its effect. Human NSCs, genetically altered to express a toxin-resistant form of EF-2 and transduced to express IL-13-PE were tested in a mouse model of glioblastoma for their ability to inhibit tumor growth $^{174}$. Administration of IL-13-PE via toxin resistant hNSCs (hNSC.IL13-PE) that were embedded in sECM and placed in the post-resection cavity of glioma bearing mice, increased MS of mice by about $60 \%{ }^{174}$.

Gain of function of Epidermal growth factor (EGF) signaling through amplification of the EGFR receptor or expression of the mutant, constitutively active form of EGFRvIII represents one of the most common and best characterized tumor specific features of glioblastoma ${ }^{175}$, extensively pursued in a variety of targeted antiglioma therapies. Antibody targeting of EGFR and EGFRvIll leads to internalization and silencing of receptor mediated signaling, inhibiting tumor growth. Preclinical studies with intratumoral delivery of MSCs modified to express a single-chain antibody against EGFRvIll on their surface, demonstrated increased survival of glioma bearing animals treated with these cells ${ }^{176}$. The enthusiasm for using EGFRvIII as therapeutic target for glioblastoma was unfortunately curbed by results of advanced clinical trials with a peptide vaccine (Rindopepimut/CDX110), developed to target EGFRvIII, results that demonstrated extensive immune 
editing following treatment with CDX110, with $82 \%$ of treated patients losing EGFRvIll expression at recurrence ${ }^{177}$. The large randomized multicenter ACT IV study with Rindopepimut for the treatment of patients with newly diagnosed EGFRvIll positive glioblastoma was discontinued as it did not meet significant OS improvements ${ }^{178}$. Failure of this trial illustrates the difficulty in designing specific therapies for glioblastoma, even when unique tumor associated antigens are identified and carefully targeted; disease progression leads to the development of a continuously moving target, increasingly difficult to manage.

\subsection{STEM CELL TRAFFICKING OF THERAPEUTIC NANOPARTICLES}

Chemotherapeutic drugs are highly toxic to normal tissues. Intratumoral administration of therapeutic agents has significant advantages over systemic administration, allowing for increased therapeutic index and concentration of the active drug in the tumor tissue, while reducing off-target effects. However, effective distribution and intratumoral retention of the drug following injection, or local placement of hydrogels or other drug-laden scaffolds is hindered by limited diffusion capacity and adverse pressure gradients that rapidly clear the drug from the tumor. Combining NSC delivery of chemotherapeutics with nanotechnology allows for more efficient distribution of drugs throughout the tumor, including to invading margins and tumor satellites, as well as decreased clearance and release of targeted agents where they are most effective.

Nanoparticles (NPs) are synthetically generated vesicles with sizes as small as $10 \mathrm{~nm}$ and as large as $1000 \mathrm{~nm}$. They are prepared from organic (lipids, polymers, dendrimers) or inorganic (metallic, ceramic) materials, can be solid or colloidal and have applications in numerous biomedical fields including theranostic applications of drug delivery, gene therapy, immunomodulation, imaging of treatment and disease progression, targeted radio- and photodynamic therapy, modulating release of bioactive molecules, minimizing toxicity and enhanced biocompatibility. Many advances have been made over the last 15 years in the field of nanotechnology and increased translation into cancer treatment is justifiably anticipated ${ }^{179}$. An excellent review of the use of NPs in brain cancer has recently been published ${ }^{180}$. In this section we will highlight preclinical studies that have employed NPs conjugated to stem cells to improve treatment of glioblastoma.

Systemic administration of NPs for cancer therapies still faces many challenges related to efficient delivery, distribution and retention in brain tumors, especially due to NP sequestration by the reticulo-edothelial system, renal clearance, transport through the BBB, overcoming the elevated intratumoral interstitial fluid pressure as well as phagocytosis by resident microglia and tumor associated macrophages. SC mediated delivery of therapeutic NPs is able to overcome these roadblocks. A proof of concept study by Roger and collaborators ${ }^{181}$ demonstrated that poly-lactic acid NPs (PLA-NPs) and lipid nanocapsules (LNCs) can be uploaded onto human BM derived MSCs and intracranially delivered into mice with established U87 orthotopic brain tumors.

NPs conjugated to NSCs have been used in an intracranial mouse model of glioblastoma, demonstrating that distribution and retention of NPs within the tumor was enhanced when NPs were surfacebound to NSCs and administered either adjacent to the tumor, in the contralateral hemisphere or 
intravenously ${ }^{182}$. These NPs were large ( 800nm, with high drug loading capacity), manufactured from polystyrene and conjugated to biotinylated NSCs (HB1.F3 cells) via streptavidin, resulting in substantial NP cargo onto the NSCs, approximately $175 \mathrm{NPS} / \mathrm{NSC}^{182}$. In a preclinical model of ovarian carcinoma, using the same human NSC line (HB1.F3), treatment of tumor-bearing animals with NSCs loaded with Silica Platinum Nanoparticles SiNP[Pt] was able to induce a higher level of [Pt] in tumors compared to treatment with NPs or drug alone ${ }^{183}$. Silica NPs loaded with Doxorubicin (DOX), named a silica nanorattle (SN), was loaded onto human BM derived MSCs using antibody (CD73, CD90) mediated conjugation, as a delayed release "time bomb" for antiglioma therapy, and tested for use in a mouse flank tumor model with U251 glioma cells ${ }^{184}$. MSC administration of DOX showed high level, broad distribution and prolonged retention compared to injection of free DOX or NP-DOX and induced increased apoptosis in U251 glioma cells.

Biotin/avidin conjugation of NSCs (HB1.F3) to Docetaxel (DTX) loaded NPs was employed in a preclinical mouse model of triple negative breast cancer ${ }^{185}$. In this study the NPs, manufactured using a $\mathrm{pH}$ responsive biotinylated polymer (PEG-PDPAEMA) were conjugated to biotinylated NSCs via an avidin linker. Acidic $\mathrm{pH}$ (6.3-6.9) is found in the extracellular space of many solid tumors, including glioblastoma ${ }^{186-189}$, making it an ideal signal for the unloading of NPs cargo. This study demonstrated that DTX loaded NPs conjugated to NSCs induced a significant decrease in tumor proliferation and vascularization after 7 days, when compared to free NP-DTX, which were rapidly cleared from the tumor ${ }^{185}$.

Doxorubicin loaded pH-sensitive mesoporous silica nanoparticles (DOX-NPs) have been tested in a preclinical intracranial glioma model on their own or when loaded onto human NSCs (HB1.F3.CD) and injected into the tumor or in the contralateral hemisphere $(5 \mathrm{~mm} \text { away })^{190}$. It was demonstrated that these NPs, when loaded onto NSCs, show a delayed release of the drug by about $20 \mathrm{hrs}$., presumably until the DOX-NPs are exposed to the acidic lysosomal compartment inside NSCs. During this time, the tumor-homing capacity of HB1.F3.CD was not compromised and they were able to reach the tumor to widely distribute and release DOX that induces the death of both tumor cells and NSCs. When compared to the intratumoral administration of loose DOX-NPs, NSCs loaded with DOX-NPs were able to significantly increase the MS of tumor-bearing mice $^{190}$.

In vitro experiments have also demonstrated that NSCs can be loaded with magnetic spinning disks (SD), 2 microns in diameter, made of several layers of cobalt, iron and boron separated by platinum and covered in gold ${ }^{191}$. In the absence of a magnetic field (MF), the SD are innocuous to the cells. Application of a rotating MF causes the disks to spin and inflict mechanical damage inside the cells. In co-culture experiments of NSCs loaded with SD and glioma cells (U87), sequential application of the magnetic field allowed for release of SDs by NSCs, which were taken up by glioma cells that were killed when the rotating MF was applied again $^{191}$.

An innovative use of NSCs as vehicles for glioma therapeutic agents was illustrated by Rachel Mooney and collaborators with the use of gold nanorods (AuNR) for plasmonic photo-thermal ablation of flank tumors in mice $^{192}$. It was demonstrated that human NSCs (HB1.F3) induced a wider, more homogeneous distribution of 
AuNR in flank tumors compared to administration of free AuNR and reduced tumor recurrence post photoablative treatment. We anticipate seeing this strategy tested in intracranial glioblastoma preclinical models.

\section{OUTLOOK FOR THE FUTURE}

Looking back at the last 20 years, the field of Stem Cell mediated delivery of antiglioma therapies into the brain has advanced considerably. Numerous preclinical studies have demonstrated the improved targeting, distribution and efficacy of SC delivery of several therapeutic agents. Enzyme/prodrug strategies with intracranial administration of NSCs producing Cytosine Deaminase or Carboxyl Esterase combined with 5-FU or Irinotecan and NSC mediated delivery of oncolytic viruses tested in early clinical trials show great promise (NCT02015819, NCT02192359, NCT03072134).

From a patient's point of view, progress is not sufficiently rapid. Just one human SC cell line (HB1.F3.CD) has been approved for clinical testing in glioblastoma, despite the fact that so many other cells, including bone marrow and adipose derived MSCs and induced NSCs have been proven effective carriers of antiglioma targeted agents in preclinical trials.

Inspired by successes in the treatment of other solid tumors with immune checkpoint inhibitors, and the hope for long-term efficacy by inducing antitumor immunological memory, extensive and sustained efforts to develop effective immunotherapy strategies for glioblastoma are much more challenging than for other cancers ${ }^{147}$. Oncolytic viruses have the proven capacity to induce immunogenic cell death (ICD) ${ }^{113}$ and can also be regarded as a form of immunotherapy. Following successful early clinical trials, many oncolytic viruses received an expedited drug review process by the FDA to be advanced to large scale randomized trials ${ }^{115}$. Study after study demonstrated that delivery of oncolytic viruses by NSCs results in enhanced tumor coverage, distribution and efficacy when compared to delivery of free virus ${ }^{131,132,137}$, advocating for efforts to fast track many more trials using this strategy into the clinic. The first-in-human clinical trial using NSCs as delivery agents for oncolytic viruses has been recently completed and results are eagerly awaited. It is anticipated that more trials with combination therapies using SC delivery of oncolytic viruses and/or immunostimulatory agents will soon be conducted. Combinatorial strategies using multimodal therapeutic SCs are likely to be more successful than current therapies. Successful targeting of glioblastoma will require combination therapy with multiple agents amenable to adaptation as the tumor progresses. Developing an expanded depository of clinically approved SCs, delivering a variety of antiglioma agents with different mechanisms of action that would be available for quick use, should bring significant improvements to currently available antiglioma therapies.

Exciting progress comes from the field of nanotechnology, with enhanced biomaterials being developed for drug and nucleic acid delivery. Conjugation of NSCs to nanoparticles (NPs) has proven to be feasible and more effective in delivering chemotherapeutics in preclinical models of glioma than direct administration of NPs. The acidic intratumoral environment brings an added bonus for $\mathrm{pH}$ responsive NPs, facilitating local 
unloading of their cargo. Great hope is provided by the ability of NPs to deliver nucleic acids into gliomas. This strategy, if optimized for use with SC mediated delivery, would overcome many current therapeutic limitations in gliomas. Targeting glioma specific genes with siRNAs or with CRISPR/Cas9 vectors represents a powerful therapeutic approach, as many of the drivers of malignant glioma and of the glioma stem cell phenotype are developmental transcription factors ${ }^{193}$, generally considered "undruggable". SCs conjugated via a pH-sensitive bond to NPs loaded with nucleic acids may provide a viable avenue to overcome current limitations. An emerging field with exciting potential therapeutic applications in glioblastoma is the ability of NSCs and MSCs to deliver proteins and nucleic acids via exosomes ${ }^{194}$.

In this review we aimed to increase awareness of the great potential and advantages brought by the use of Stem Cells as vehicles for efficient and effective delivery of targeted anti-glioma therapies. At the same time, we emphasized the importance of critical experimental parameters, especially regarding the number of effectively distributed intratumoral SCs needed to ensure therapeutic efficacy. There is a great need to develop more clinically approved SCs for glioblastoma therapy and good practice manufacturing protocols that would make sufficient cells available for therapeutic use. This entails dedicated and diligent work to demonstrate and document the safety of these cellular therapeutic agents and a push for regulatory approval. Treatment failure in glioblastoma is in large part due to limitations in the delivery of therapeutic agents into invasive intracranial tumors. Stem Cells represent a viable solution for this problem. Why wait?

Acknowledgements: We thank Dr. Karin Muraszko, Chair and Julian T. Hoff Professor of Neurosurgical Surgery and Dr. Richard Keep, Director Crosby Neurosurgical Laboratories for their help and support. We also thank the Undergraduate Research Opportunity Program at the University of Michigan for supporting the work of MK and ZS. Grant support was provided by NIH/NINDS R21NS107879 (AC). 


\section{REFERENCES}

1. Breunig, J.J., Haydar, T.F. \& Rakic, P. Neural stem cells: historical perspective and future prospects. Neuron 70, 614-625 (2011).

2. His, W. Die Entwickelung des menschlichen Gehirns: während der ersten Monate, (S. Hirzel, 1904).

3. Gage, F.H., Ray, J. \& Fisher, L.J. Isolation, characterization, and use of stem cells from the CNS. Annual review of neuroscience 18, 159-192 (1995).

4. Weiss, S., et al. Is there a neural stem cell in the mammalian forebrain? Trends in neurosciences 19, 387-393 (1996).

5. Temple, S. Division and differentiation of isolated CNS blast cells in microculture. Nature 340, 471-473 (1989).

6. Temple, S. The development of neural stem cells. Nature 414, 112-117 (2001).

7. Snyder, E.Y., Taylor, R.M. \& Wolfe, J.H. Neural progenitor cell engraftment corrects lysosomal storage throughout the MRS VII mouse brain. Nature 374, 367-370 (1995).

8. $\quad$ Armstrong, R.J., Watts, C., Svendsen, C.N., Dunnett, S.B. \& Rosser, A.E. Survival, neuronal differentiation, and fiber outgrowth of propagated human neural precursor grafts in an animal model of Huntington's disease. Cell transplantation 9, 55-64 (2000).

9. Svendsen, C.N., et al. Long-term survival of human central nervous system progenitor cells transplanted into a rat model of Parkinson's disease. Experimental neurology 148, 135-146 (1997).

10. Olanow, C. \& Fahn, S. Fetal nigral transplantation as a therapy for Parkinson's disease. in Restorative therapies in Parkinson's disease 93-118 (Springer, 2006).

11. Yandava, B.D., Billinghurst, L.L. \& Snyder, E.Y. "Global" cell replacement is feasible via neural stem cell transplantation: evidence from the dysmyelinated shiverer mouse brain. Proceedings of the National Academy of Sciences 96, 7029-7034 (1999).

12. Silani, V., Cova, L., Corbo, M., Ciammola, A. \& Polli, E. Stem-cell therapy for amyotrophic lateral sclerosis. The Lancet 364, 200-202 (2004).

13. Teng, Y.D., et al. Functional recovery following traumatic spinal cord injury mediated by a unique polymer scaffold seeded with neural stem cells. Proceedings of the National Academy of Sciences 99 , 3024-3029 (2002).

14. Bauer, G., Elsallab, M. \& Abou-El-Enein, M. Concise Review: A comprehensive analysis of reported adverse events in patients receiving unproven stem cell-based interventions. Stem cells translational medicine 7, 676-685 (2018).

15. Aboody, K., Capela, A., Niazi, N., Stern, J.H. \& Temple, S. Translating stem cell studies to the clinic for CNS repair: current state of the art and the need for a Rosetta stone. Neuron 70, 597-613 (2011).

16. Ostrom, Q.T., Cote, D.J., Ascha, M., Kruchko, C. \& Barnholtz-Sloan, J.S. Adult glioma incidence and survival by race or ethnicity in the United States from 2000 to 2014. JAMA oncology 4, 1254-1262 (2018).

17. Stupp, R., et al. Radiotherapy plus concomitant and adjuvant temozolomide for glioblastoma. New England Journal of Medicine 352, 987-996 (2005).

18. Osuka, S. \& Van Meir, E.G. Overcoming therapeutic resistance in glioblastoma: the way forward. The Journal of clinical investigation 127, 415-426 (2017).

19. Singh, S.K., et al. Identification of a cancer stem cell in human brain tumors. Cancer research 63, 5821 5828 (2003).

20. Ligon, K.L., et al. Olig2-regulated lineage-restricted pathway controls replication competence in neural stem cells and malignant glioma. Neuron 53, 503-517 (2007).

21. Bao, S., et al. Targeting cancer stem cells through L1CAM suppresses glioma growth. Cancer research 68, 6043-6048 (2008).

22. Beier, D., Schulz, J.B. \& Beier, C.P. Chemoresistance of glioblastoma cancer stem cells-much more complex than expected. Molecular cancer 10, 128 (2011).

23. Calinescu, A.A., et al. Survival and proliferation of neural progenitor derived glioblastomas under hypoxic stress is controlled by a CXCL12/CXCR4 autocrine positive feedback mechanism. Clin Cancer $\operatorname{Res}(2016)$.

24. Sanai, N., Alvarez-Buylla, A. \& Berger, M.S. Neural stem cells and the origin of gliomas. New England Journal of Medicine 353, 811-822 (2005). 
25. Lim, D.A., et al. Relationship of glioblastoma multiforme to neural stem cell regions predicts invasive and multifocal tumor phenotype. Neuro-oncology 9, 424-429 (2007).

26. Rakic, P. \& Lombroso, P.J. Development of the cerebral cortex: I. Forming the cortical structure. Journal of the American Academy of Child \& Adolescent Psychiatry 37, 116-117 (1998).

27. Noctor, S.C., Flint, A.C., Weissman, T.A., Dammerman, R.S. \& Kriegstein, A.R. Neurons derived from radial glial cells establish radial units in neocortex. Nature 409, 714-720 (2001).

28. Kriegstein, A. \& Alvarez-Buylla, A. The glial nature of embryonic and adult neural stem cells. Annual review of neuroscience 32, 149-184 (2009).

29. Emsley, J.G., Mitchell, B.D., Kempermann, G. \& Macklis, J.D. Adult neurogenesis and repair of the adult CNS with neural progenitors, precursors, and stem cells. Progress in neurobiology 75, 321-341 (2005).

30. Reynolds, B.A. \& Rietze, R.L. Neural stem cells and neurospheres-re-evaluating the relationship. Nature methods 2, 333-336 (2005).

31. Wakeman, D.R., Hofmann, M.R., Teng, Y.D. \& Snyder, E.Y. Neural progenitors. in Human Adult Stem Cells 1-44 (Springer, 2009).

32. Horiguchi, S., et al. Neural precursor cells derived from human embryonic brain retain regional specificity. Journal of neuroscience research 75, 817-824 (2004).

33. Bhaduri, A., et al. Outer Radial Glia-like Cancer Stem Cells Contribute to Heterogeneity of Glioblastoma. Cell Stem Cell 26, 48-63. e46 (2020).

34. Darmanis, S., et al. Single-cell RNA-seq analysis of infiltrating neoplastic cells at the migrating front of human glioblastoma. Cell reports 21, 1399-1410 (2017).

35. Venteicher, A.S., et al. Decoupling genetics, lineages, and microenvironment in IDH-mutant gliomas by single-cell RNA-seq. Science 355(2017).

36. Filbin, M.G., et al. Developmental and oncogenic programs in H3K27M gliomas dissected by single-cell RNA-seq. Science 360, 331-335 (2018).

37. Neftel, C., et al. An integrative model of cellular states, plasticity, and genetics for glioblastoma. Cell 178, 835-849. e821 (2019).

38. Tiveron, M.-C. \& Cremer, H. CXCL12/CXCR4 signalling in neuronal cell migration. Current opinion in neurobiology 18, 237-244 (2008).

39. Goffart, N., et al. Adult mouse subventricular zones stimulate glioblastoma stem cells specific invasion through CXCL12/CXCR4 signaling. Neuro-oncology 17, 81-94 (2015).

40. Morrison, S.J. \& Scadden, D.T. The bone marrow niche for haematopoietic stem cells. Nature 505, 327-334 (2014).

41. Tabatabai, G., Frank, B., Möhle, R., Weller, M. \& Wick, W. Irradiation and hypoxia promote homing of haematopoietic progenitor cells towards gliomas by TGF- $\beta$-dependent HIF-1 $\alpha$-mediated induction of CXCL12. Brain 129, 2426-2435 (2006).

42. Komatani, H., Sugita, Y., Arakawa, F., Ohshima, K. \& Shigemori, M. Expression of CXCL12 on pseudopalisading cells and proliferating microvessels in glioblastomas: an accelerated growth factor in glioblastomas. International journal of oncology 34, 665-672 (2009).

43. Inukai, M., et al. Hypoxia-mediated cancer stem cells in pseudopalisades with activation of hypoxiainducible factor-1a/Akt axis in glioblastoma. Human pathology 46, 1496-1505 (2015).

44. McKay, R. Stem cells in the central nervous system. Science 276, 66-71 (1997).

45. Gage, F.H. Mammalian neural stem cells. Science 287, 1433-1438 (2000).

46. Flax, J.D., et al. Engraftable human neural stem cells respond to development cues, replace neurons, and express foreign genes. Nature biotechnology 16, 1033-1039 (1998).

47. Martinez-Serrano, A., Lundberg, C. \& Björklund, A. Use of conditionally immortalized neural progenitors for transplantation and gene transfer to the CNS. in Isolation, Characterization and Utilization of CNS Stem Cells 151-168 (Springer, 1997).

48. Snyder, E.Y. Neural stem-like cells: developmental lessons with therapeutic potential. The Neuroscientist 4, 408-425 (1998).

49. Aboody, K.S., et al. Neural stem cells display extensive tropism for pathology in adult brain: evidence from intracranial gliomas. Proceedings of the National Academy of Sciences 97, 12846-12851 (2000).

50. Ryder, E.F., Snyder, E.Y. \& Cepko, C.L. Establishment and characterization of multipotent neural cell lines using retrovirus vector-mediated oncogene transfer. Journal of neurobiology 21, 356-375 (1990). 
51. Kim, S.U. Human neural stem cells genetically modified for brain repair in neurological disorders. Neuropathology 24, 159-171 (2004).

52. Ryu, J.K., et al. Adenosine triphosphate induces proliferation of human neural stem cells: Role of calcium and p70 ribosomal protein S6 kinase. Journal of neuroscience research 72, 352-362 (2003).

53. Aboody, K.S., et al. Neural stem cell-mediated enzyme/prodrug therapy for glioma: Preclinical studies. Science translational medicine 5, 184ra159-184ra159 (2013).

54. Donato, R., et al. Differential development of neuronal physiological responsiveness in two human neural stem cell lines. BMC neuroscience 8, 36 (2007).

55. Ostenfeld, T., et al. Regional specification of rodent and human neurospheres. Developmental brain research 134, 43-55 (2002).

56. Kelly, T.K., Karsten, S.L., Geschwind, D.H. \& Kornblum, H.I. Cell lineage and regional identity of cultured spinal cord neural stem cells and comparison to brain-derived neural stem cells. PloS one 4, e4213 (2009).

57. Hack, M., Sugimori, M., Lundberg, C., Nakafuku, M. \& Götz, M. Regionalization and fate specification in neurospheres: the role of Olig2 and Pax6. Molecular and Cellular Neuroscience 25, 664-678 (2004).

58. Santa-Olalla, J., Baizabal, J.M., Fregoso, M., del Carmen Cardenas, M. \& Covarrubias, L. The in vivo positional identity gene expression code is not preserved in neural stem cells grown in culture. European Journal of Neuroscience 18, 1073-1084 (2003).

59. Chamberlain, G., Fox, J., Ashton, B. \& Middleton, J. Concise review: mesenchymal stem cells: their phenotype, differentiation capacity, immunological features, and potential for homing. Stem cells 25, 2739-2749 (2007).

60. Klopp, A.H., Gupta, A., Spaeth, E., Andreeff, M. \& Marini III, F. Concise review: dissecting a discrepancy in the literature: do mesenchymal stem cells support or suppress tumor growth? Stem cells 29, 11-19 (2011).

61. Ahmed, A.U., et al. A comparative study of neural and mesenchymal stem cell-based carriers for oncolytic adenovirus in a model of malignant glioma. Molecular pharmaceutics 8, 1559-1572 (2011).

62. Ahmed, A.U., et al. A preclinical evaluation of neural stem cell-based cell carrier for targeted antiglioma oncolytic virotherapy. Journal of the National Cancer Institute 105, 968-977 (2013).

63. Amariglio, N., et al. Donor-derived brain tumor following neural stem cell transplantation in an ataxia telangiectasia patient. PLoS Med 6, e1000029 (2009).

64. Berkowitz, A.L., et al. Glioproliferative lesion of the spinal cord as a complication of" stem-cell tourism". The New England journal of medicine 375, 196-198 (2016).

65. Bagó, J.R., et al. Therapeutically engineered induced neural stem cells are tumour-homing and inhibit progression of glioblastoma. Nature communications 7, 10593 (2016).

66. Bagó, J.R., et al. Tumor-homing cytotoxic human induced neural stem cells for cancer therapy. Science translational medicine 9, eaah6510 (2017).

67. Gao, M., et al. Tumourigenicity and immunogenicity of induced neural stem cell grafts versus induced pluripotent stem cell grafts in syngeneic mouse brain. Scientific reports 6, 1-13 (2016).

68. Lettry, V., Hagler, S.B., Khagi, S. \& Hingtgen, S.D. Tumor-homing Stem Cell Therapy for Brain Cancer. Current Surgery Reports 5(2017).

69. Herrlinger, U., et al. Neural precursor cells for delivery of replication-conditional HSV-1 vectors to intracerebral gliomas. Molecular Therapy 1, 347-357 (2000).

70. Benedetti, S., et al. Gene therapy of experimental brain tumors using neural progenitor cells. Nature medicine 6, 447-450 (2000).

71. Ehtesham, M., et al. Induction of glioblastoma apoptosis using neural stem cell-mediated delivery of tumor necrosis factor-related apoptosis-inducing ligand. Cancer research 62, 7170-7174 (2002).

72. Moolten, F.L. Tumor chemosensitivity conferred by inserted herpes thymidine kinase genes: paradigm for a prospective cancer control strategy. Cancer research 46, 5276-5281 (1986).

73. Hossain, J.A., Marchini, A., Fehse, B., Bjerkvig, R. \& Miletic, H. Suicide gene therapy for the treatment of high-grade glioma: past lessons, present trends, and future prospects. Neuro-Oncology Advances 2, vdaa013 (2020).

74. Uhl, M., et al. Migratory neural stem cells for improved thymidine kinase-based gene therapy of malignant gliomas. Biochemical and biophysical research communications 328, 125-129 (2005). 
75. Li, S., et al. Bystander effect-mediated gene therapy of gliomas using genetically engineered neural stem cells. Cancer Gene Therapy 12, 600-607 (2005).

76. Li, S., et al. Bystander effect in glioma suicide gene therapy using bone marrow stromal cells. Stem Cell Research 9, 270-276 (2012).

77. Kim, S.-K., et al. Human neural stem cells target experimental intracranial medulloblastoma and deliver a therapeutic gene leading to tumor regression. Clinical Cancer Research 12, 5550-5556 (2006).

78. Altanerova, V., et al. Human adipose tissue-derived mesenchymal stem cells expressing yeast cytosinedeaminase:: uracil phosphoribosyltransferase inhibit intracerebral rat glioblastoma. International journal of cancer 130, 2455-2463 (2012).

79. van Kuilenburg, A.B. Dihydropyrimidine dehydrogenase and the efficacy and toxicity of 5-fluorouracil. European journal of cancer 40, 939-950 (2004).

80. Chung, T., et al. Dihydropyrimidine dehydrogenase is a prognostic marker for mesenchymal stem cellmediated cytosine deaminase gene and 5-fluorocytosine prodrug therapy for the treatment of recurrent gliomas. Theranostics 6, 1477 (2016).

81. Kim, S.U., et al. Production and characterization of immortal human neural stem cell line with multipotent differentiation property. in Neural Stem Cells 103-121 (Springer, 2008).

82. Portnow, J., et al. A first-in-human study of neural stem cells (NSCs) expressing cytosine deaminase (CD) in combination with 5-fluorocytosine (5-FC) in patients with recurrent high-grade glioma. (American Society of Clinical Oncology, 2013).

83. Portnow, J., et al. Neural stem cell-based anticancer gene therapy: a first-in-human study in recurrent high-grade glioma patients. Clinical Cancer Research 23, 2951-2960 (2017).

84. Choi, S.A., et al. Human adipose tissue-derived mesenchymal stem cells: characteristics and therapeutic potential as cellular vehicles for prodrug gene therapy against brainstem gliomas. European Journal of Cancer 48, 129-137 (2012).

85. Yin, J., et al. hMSC-mediated concurrent delivery of endostatin and carboxylesterase to mouse xenografts suppresses glioma initiation and recurrence. Molecular Therapy 19, 1161-1169 (2011).

86. Gutova, M., et al. Neural stem cell-mediated CE/CPT-11 enzyme/prodrug therapy in transgenic mouse model of intracerebellar medulloblastoma. Gene therapy 20, 143-150 (2013).

87. Wierdl, M., et al. An improved human carboxylesterase for enzyme/prodrug therapy with CPT-11. Cancer gene therapy 15, 183-192 (2008).

88. Metz, M.Z., et al. Neural stem cell-mediated delivery of irinotecan-activating carboxylesterases to glioma: Implications for clinical use. Stem cells translational medicine 2, 983-992 (2013).

89. Gutova, M., et al. Quantitative evaluation of intraventricular delivery of therapeutic neural stem cells to orthotopic glioma. Frontiers in oncology 9, 68 (2019).

90. Barish, M.E., et al. Human neural stem cell biodistribution and predicted tumor coverage by a diffusible therapeutic in a mouse glioma model. Stem cells translational medicine 6, 1522-1532 (2017).

91. Ghoda, L., et al. STEM-32. Preclinical Safety and efficacy studies of Neural Stem Cell mediated carboxylesterase/irinotecan enzyme/prodrug gene therapy for recurrent glioma leading to first in human phase I clinical trial. Neuro-oncology 19, vi232-vi233 (2017).

92. Levine, A.J. p53: 800 million years of evolution and 40 years of discovery. Nature Reviews Cancer, 110 (2020).

93. Network, C.G.A.R. Comprehensive genomic characterization defines human glioblastoma genes and core pathways. Nature 455, 1061 (2008).

94. Von Karstedt, S., Montinaro, A. \& Walczak, H. Exploring the TRAILs less travelled: TRAIL in cancer biology and therapy. Nature Reviews Cancer 17, 352 (2017).

95. Micheau, O., Shirley, S. \& Dufour, F. Death receptors as targets in cancer. British journal of pharmacology 169, 1723-1744 (2013).

96. Ashkenazi, A., et al. Safety and antitumor activity of recombinant soluble Apo2 ligand. The Journal of clinical investigation 104, 155-162 (1999).

97. Belkahla, H., et al. TRAIL-NP hybrids for cancer therapy: a review. Nanoscale 9, 5755-5768 (2017).

98. Dörr, J., Waiczies, S., Wendling, U., Seeger, B. \& Zipp, F. Induction of TRAIL-mediated glioma cell death by human T cells. Journal of neuroimmunology 122, 117-124 (2002). 
99. Corsten, M.F., et al. MicroRNA-21 knockdown disrupts glioma growth in vivo and displays synergistic cytotoxicity with neural precursor cell-delivered S-TRAIL in human gliomas. Cancer research 67, 89949000 (2007).

100. Chan, J.A., Krichevsky, A.M. \& Kosik, K.S. MicroRNA-21 is an antiapoptotic factor in human glioblastoma cells. Cancer research 65, 6029-6033 (2005).

101. Hingtgen, S., et al. Targeting multiple pathways in gliomas with stem cell and viral delivered S-TRAIL and Temozolomide. Molecular cancer therapeutics 7, 3575-3585 (2008).

102. Lashinger, L.M., et al. Bortezomib abolishes tumor necrosis factor-related apoptosis-inducing ligand resistance via a p21-dependent mechanism in human bladder and prostate cancer cells. Cancer research 65, 4902-4908 (2005).

103. Koschny, R., et al. Bortezomib Sensitizes Primary Human Astrocytoma Cells of WHO Grades I to IV for Tumor Necrosis Factor-Related Apoptosis-Inducing Ligand-Induced Apoptosis. Clinical Cancer Research 13, 3403-3412 (2007).

104. Balyasnikova, I.V., Ferguson, S.D., Han, Y., Liu, F. \& Lesniak, M.S. Therapeutic effect of neural stem cells expressing TRAIL and bortezomib in mice with glioma xenografts. Cancer letters 310, 148-159 (2011).

105. Perlstein, B., et al. TRAIL conjugated to nanoparticles exhibits increased anti-tumor activities in glioma cells and glioma stem cells in vitro and in vivo. Neuro-oncology 15, 29-40 (2013).

106. Jiang, X., et al. Nanoparticle engineered TRAIL-overexpressing adipose-derived stem cells target and eradicate glioblastoma via intracranial delivery. Proceedings of the National Academy of Sciences 113, 13857-13862 (2016).

107. Norton, L. \& Massagué, J. Is cancer a disease of self-seeding? Nature medicine 12, 875-878 (2006).

108. Ehtesham, M., Winston, J., Kabos, P. \& Thompson, R. CXCR4 expression mediates glioma cell invasiveness. Oncogene 25, 2801-2806 (2006).

109. Liu, Z., Kuang, W., Zhou, Q. \& Zhang, Y. TGF- $\beta 1$ secreted by M2 phenotype macrophages enhances the stemness and migration of glioma cells via the SMAD2/3 signalling pathway. International Journal of Molecular Medicine 42, 3395-3403 (2018).

110. Reinshagen, C., et al. CRISPR-enhanced engineering of therapy-sensitive cancer cells for selftargeting of primary and metastatic tumors. Science translational medicine 10(2018).

111. Kaufmann, J.K. \& Chiocca, E.A. Glioma virus therapies between bench and bedside. Neuro-oncology 16, 334-351 (2014).

112. Wollmann, G., Ozduman, K. \& van den Pol, A.N. Oncolytic virus therapy of glioblastoma multiformeconcepts and candidates. Cancer journal (Sudbury, Mass.) 18, 69 (2012).

113. Chiocca, E.A. \& Rabkin, S.D. Oncolytic viruses and their application to cancer immunotherapy. Cancer immunology research 2, 295-300 (2014).

114. Kelly, E. \& Russell, S.J. History of oncolytic viruses: genesis to genetic engineering. Molecular Therapy 15, 651-659 (2007).

115. Martikainen, M. \& Essand, M. Virus-based immunotherapy of glioblastoma. Cancers 11, 186 (2019).

116. Desjardins, A., et al. Recurrent glioblastoma treated with recombinant poliovirus. New England journal of medicine 379, 150-161 (2018).

117. Desjardins, A., et al. Oncolytic polio/rhinovirus recombinant (PVSRIPO) against WHO grade IV malignant glioma (MG): Experience with retreatment of survivors from the phase I trial. Journal of Clinical Oncology 37, 2060-2060 (2019).

118. Cloughesy, T.F., et al. Durable complete responses in some recurrent high-grade glioma patients treated with Toca 511+ Toca FC. Neuro-oncology 20, 1383-1392 (2018).

119. Lang, F.F., et al. Phase I study of DNX-2401 (Delta-24-RGD) oncolytic adenovirus: replication and immunotherapeutic effects in recurrent malignant glioma. Journal of Clinical Oncology 36, 1419 (2018).

120. Cloughesy, T., et al. LTBK-08. TOCA 511 \&amp; TOCA FC VERSUS STANDARD OF CARE IN PATIENTS WITH RECURRENT HIGH GRADE GLIOMA. Neuro-Oncology 21, vi284-vi284 (2019).

121. Todo, T., Martuza, R.L., Rabkin, S.D. \& Johnson, P.A. Oncolytic herpes simplex virus vector with enhanced MHC class I presentation and tumor cell killing. Proceedings of the National Academy of Sciences 98, 6396-6401 (2001).

122. Todo, T. ATIM-14. RESULTS OF PHASE II CLINICAL TRIAL OF ONCOLYTIC HERPES VIRUS G47D IN PATIENTS WITH GLIOBLASTOMA. Neuro-Oncology 21, vi4-vi4 (2019). 
123. Foreman, P.M., Friedman, G.K., Cassady, K.A. \& Markert, J.M. Oncolytic virotherapy for the treatment of malignant glioma. Neurotherapeutics 14, 333-344 (2017).

124. Chiocca, E.A., et al. A phase I open-label, dose-escalation, multi-institutional trial of injection with an E1B-Attenuated adenovirus, ONYX-015, into the peritumoral region of recurrent malignant gliomas, in the adjuvant setting. Molecular Therapy 10, 958-966 (2004).

125. Fueyo, J., et al. A mutant oncolytic adenovirus targeting the Rb pathway produces anti-glioma effect in vivo. Oncogene 19, 2-12 (2000).

126. O'Shea, C.C., et al. Late viral RNA export, rather than p53 inactivation, determines ONYX-015 tumor selectivity. Cancer cell 6, 611-623 (2004).

127. Rojas, J.J., et al. Minimal RB-responsive E1A promoter modification to attain potency, selectivity, and transgene-arming capacity in oncolytic adenoviruses. Molecular Therapy 18, 1960-1971 (2010).

128. Guedán Carrió, S., Gros Vidal, A., Mercadé Gil, M.E., Cascalló Piqueras, M. \& Alemany Bonastre, R. Hyaluronidase expression by an oncolytic adenovirus enhances its intratumoral spread and supresses tumor growth. Molecular Therapy, 2010, vol. 18, num. 7, p. 1275-1283 (2010).

129. Rodríguez-García, A., et al. Safety and efficacy of VCN-01, an oncolytic adenovirus combining fiber HSG-binding domain replacement with RGD and hyaluronidase expression. Clinical Cancer Research 21, 1406-1418 (2015).

130. Vera, B., et al. Characterization of the antiglioma effect of the oncolytic adenovirus VCN-01. PLoS One 11, e0147211 (2016).

131. Martinez-Quintanilla, J., He, D., Wakimoto, H., Alemany, R. \& Shah, K. Encapsulated stem cells loaded with hyaluronidase-expressing oncolytic virus for brain tumor therapy. Molecular therapy 23, 108-118 (2015).

132. Duebgen, M., et al. Stem cells loaded with multimechanistic oncolytic herpes simplex virus variants for brain tumor therapy. JNCl: Journal of the National Cancer Institute 106(2014).

133. Josiah, D.T., et al. Adipose-derived stem cells as therapeutic delivery vehicles of an oncolytic virus for glioblastoma. Molecular Therapy 18, 377-385 (2010).

134. Sonabend, A.M., et al. Mesenchymal stem cells effectively deliver an oncolytic adenovirus to intracranial glioma. Stem cells 26, 831-841 (2008).

135. Warrier, N.M., Agarwal, P. \& Kumar, P. Emerging Importance of Survivin in Stem Cells and Cancer: the Development of New Cancer Therapeutics. Stem Cell Reviews and Reports, 1-25 (2020).

136. Tyler, M.A., et al. Neural stem cells target intracranial glioma to deliver an oncolytic adenovirus in vivo. Gene therapy 16, 262-278 (2009).

137. Ahmed, A.U., et al. Neural stem cell-based cell carriers enhance therapeutic efficacy of an oncolytic adenovirus in an orthotopic mouse model of human glioblastoma. Molecular Therapy 19, 1714-1726 (2011).

138. Li, G., Bonamici, N., Dey, M., Lesniak, M.S. \& Balyasnikova, I.V. Intranasal Delivery of Stem Cell-based Therapies for the Treatment of Brain Malignancies. Expert Opinion on Drug Delivery (2017).

139. Zhao, D., et al. Neural stem cell tropism to glioma: critical role of tumor hypoxia. Molecular Cancer Research 6, 1819-1829 (2008).

140. Dey, M., et al. Intranasal Oncolytic Virotherapy with CXCR4-Enhanced Stem Cells Extends Survival in Mouse Model of Glioma. Stem cell reports 7, 471-482 (2016).

141. Tobias, A.L., et al. The timing of neural stem cell-based virotherapy is critical for optimal therapeutic efficacy when applied with radiation and chemotherapy for the treatment of glioblastoma. Stem cells translational medicine 2, 655-666 (2013).

142. Spencer, D., et al. Pharmacologic modulation of nasal epithelium augments neural stem cell targeting of glioblastoma. Theranostics 9, 2071 (2019).

143. Lin, D., et al. Novel method for visualizing and modeling the spatial distribution of neural stem cells within intracranial glioma. Neuroimage 37, S18-S26 (2007).

144. Morshed, R.A., et al. Analysis of glioblastoma tumor coverage by oncolytic virus-loaded neural stem cells using MRI-based tracking and histological reconstruction. Cancer gene therapy 22, 55-61 (2015).

145. Thaci, B., et al. Pharmacokinetic study of neural stem cell-based cell carrier for oncolytic virotherapy: targeted delivery of the therapeutic payload in an orthotopic brain tumor model. Cancer gene therapy 19, 431-442 (2012). 
146. Lesniak, M.S., et al. ACTR-36. A PHASE I STUDY OF NEURAL STEM CELLS LOADED WITH AN ONCOLYTIC ADENOVIRUS FOR PATIENTS WITH NEWLY DIAGNOSED MALIGNANT GLIOMA: PRELIMINARY SAFETY AND DATA ANALYSIS. Neuro-oncology 19, vi8 (2017).

147. Fecci, P.E. \& Sampson, J.H. The current state of immunotherapy for gliomas: an eye toward the future: JNSPG 75th Anniversary Invited Review Article. Journal of neurosurgery 131, 657-666 (2019).

148. Calinescu, A.-A., et al. Overview of current immunotherapeutic strategies for glioma. Immunotherapy 7 , 1073-1104 (2015).

149. Okada, H. \& Pollack, I.F. Cytokine gene therapy for malignant glioma. Expert opinion on biological therapy 4, 1609-1620 (2004).

150. Sikorski, C.W. \& Lesniak, M.S. Immunotherapy for malignant glioma: current approaches and future directions. Neurological research 27, 703-716 (2005).

151. Tepper, R.I., Pattengale, P.K. \& Leder, P. Murine interleukin-4 displays potent anti-tumor activity in vivo. Cell 57, 503-512 (1989).

152. Okada, H., Banchereau, J. \& Lotze, M.T. Interleukin-4. The cytokine handbook 1, 227-262 (2003).

153. Joshi, B.H., et al. In situ expression of interleukin-4 (IL-4) receptors in human brain tumors and cytotoxicity of a recombinant IL-4 cytotoxin in primary glioblastoma cell cultures. Cancer Research 61, 8058-8061 (2001).

154. Brunda, M.J., et al. Antitumor and antimetastatic activity of interleukin 12 against murine tumors. The Journal of experimental medicine 178, 1223-1230 (1993).

155. Colombo, M.P. \& Trinchieri, G. Interleukin-12 in anti-tumor immunity and immunotherapy. Cytokine \& growth factor reviews 13, 155-168 (2002).

156. Ehtesham, M., et al. The use of interleukin 12-secreting neural stem cells for the treatment of intracranial glioma. Cancer research 62, 5657-5663 (2002).

157. Yang, S.-Y., Liu, H. \& Zhang, J.-N. Gene therapy of rat malignant gliomas using neural stem cells expressing IL-12. DNA and cell biology 23, 381-389 (2004).

158. Hong, X., Miller, C., Savant-Bhonsale, S. \& Kalkanis, S.N. Antitumor treatment using interleukin-12secreting marrow stromal cells in an invasive glioma model. Neurosurgery 64, 1139-1147 (2009).

159. Ryu, C.H., et al. Gene therapy of intracranial glioma using interleukin 12-secreting human umbilical cord blood-derived mesenchymal stem cells. Human gene therapy 22, 733-743 (2011).

160. Yuan, X., Hu, J., Belladonna, M.L., Black, K.L. \& John, S.Y. Interleukin-23-Expressing Bone MarrowDerived Neural Stem-Like Cells Exhibit Antitumor Activity against Intracranial Glioma. Cancer research 66, 2630-2638 (2006).

161. Xu, G., et al. Adenoviral-mediated interleukin-18 expression in mesenchymal stem cells effectively suppresses the growth of glioma in rats. Cell biology international 33, 466-474 (2009).

162. Xu, G., Guo, Y., Seng, Z., Cui, G. \& Qu, J. Bone marrow-derived mesenchymal stem cells co-expressing interleukin-18 and interferon- $\beta$ exhibit potent antitumor effect against intracranial glioma in rats. Oncology Reports 34, 1915-1922 (2015).

163. Gunnarsson, S., et al. Intratumoral IL-7 delivery by mesenchymal stromal cells potentiates IFNYtransduced tumor cell immunotherapy of experimental glioma. Journal of neuroimmunology 218, 140144 (2010).

164. Choi, S.H., et al. Tumor resection recruits effector T cells and boosts therapeutic efficacy of encapsulated stem cells expressing IFN $\beta$ in glioblastomas. Clinical Cancer Research 23, 7047-7058 (2017).

165. Dent, P., et al. MDA-7/IL-24 as a cancer therapeutic: from bench to bedside. Anti-cancer drugs 21, 725 (2010).

166. Hingtgen, S., et al. A first-generation multi-functional cytokine for simultaneous optical tracking and tumor therapy. PloS one 7(2012).

167. Piccirillo, S., et al. Bone morphogenetic proteins inhibit the tumorigenic potential of human brain tumour-initiating cells. Nature 444, 761-765 (2006).

168. Li, Q., et al. Mesenchymal stem cells from human fat engineered to secrete BMP4 are nononcogenic, suppress brain cancer, and prolong survival. Clinical Cancer Research 20, 2375-2387 (2014).

169. Mangraviti, A., et al. Non-virally engineered human adipose mesenchymal stem cells produce BMP4, target brain tumors, and extend survival. Biomaterials 100, 53-66 (2016). 
170. Cavaillon, J.-M. Historical links between toxinology and immunology. Pathogens and disease 76, fty019 (2018).

171. Debinski, W., Obiri, N.I., Powers, S.K., Pastan, I. \& Puri, R.K. Human glioma cells overexpress receptors for interleukin 13 and are extremely sensitive to a novel chimeric protein composed of interleukin 13 and pseudomonas exotoxin. Clinical Cancer Research 1, 1253-1258 (1995).

172. Thaci, B., et al. Significance of interleukin-13 receptor alpha 2-targeted glioblastoma therapy. Neurooncology 16, 1304-1312 (2014).

173. Kunwar, S., et al. Phase III randomized trial of CED of IL13-PE38QQR vs Gliadel wafers for recurrent glioblastoma. Neuro-oncology 12, 871-881 (2010).

174. Stuckey, D.W., Hingtgen, S.D., Karakas, N., Rich, B.E. \& Shah, K. Engineering toxin-resistant therapeutic stem cells to treat brain tumors. Stem cells 33, 589-600 (2015).

175. Heimberger, A.B., Suki, D., Yang, D., Shi, W. \& Aldape, K. The natural history of EGFR and EGFRvIll in glioblastoma patients. Journal of translational medicine 3, 1-6 (2005).

176. Balyasnikova, I.V., Ferguson, S.D., Sengupta, S., Han, Y. \& Lesniak, M.S. Mesenchymal stem cells modified with a single-chain antibody against EGFRvIll successfully inhibit the growth of human xenograft malignant glioma. PloS one 5, e9750 (2010).

177. Babu, R. \& Adamson, D.C. Rindopepimut: an evidence-based review of its therapeutic potential in the treatment of EGFRvIII-positive glioblastoma. Core evidence 7, 93 (2012).

178. Gerstner, E.R. ACT IV: the final act for rindopepimut? The Lancet Oncology 18, 1294-1296 (2017).

179. Martins, J.P., et al. The solid progress of nanomedicine. Drug Delivery and Translational Research, 1-4 (2020).

180. Bhargav, A.G., Mondal, S.K., Garcia, C.A., Green, J.J. \& Quiñones-Hinojosa, A. Nanomedicine Revisited: Next Generation Therapies for Brain Cancer. Advanced Therapeutics, 2000118 (2020).

181. Roger, M., et al. Mesenchymal stem cells as cellular vehicles for delivery of nanoparticles to brain tumors. Biomaterials 31, 8393-8401 (2010).

182. Mooney, R., et al. Neural stem cells improve intracranial nanoparticle retention and tumor-selective distribution. Future oncology 10, 401-415 (2014).

183. Cao, P., et al. Intraperitoneal administration of neural stem cell-nanoparticle conjugates targets chemotherapy to ovarian tumors. Bioconjugate chemistry 28, 1767-1776 (2017).

184. Li, L., et al. Silica nanorattle-doxorubicin-anchored mesenchymal stem cells for tumor-tropic therapy. ACS nano 5, 7462-7470 (2011).

185. Mooney, R., et al. Conjugation of pH-responsive nanoparticles to neural stem cells improves intratumoral therapy. Journal of controlled release 191, 82-89 (2014).

186. Filatova, A., et al. Acidosis acts through HSP90 in a PHD/VHL-independent manner to promote HIF function and stem cell maintenance in glioma. Cancer research 76, 5845-5856 (2016).

187. Gillies, R.J., Raghunand, N., Garcia-Martin, M.L. \& Gatenby, R.A. pH imaging. IEEE Engineering in medicine and biology magazine 23, 57-64 (2004).

188. Corbet, C. \& Feron, O. Tumour acidosis: from the passenger to the driver's seat. Nature Reviews Cancer 17, 577 (2017).

189. Hjelmeland, A.B., et al. Acidic stress promotes a glioma stem cell phenotype. Cell Death \& Differentiation 18, 829-840 (2011).

190. Cheng, Y., et al. Nanoparticle-Programmed Self-Destructive Neural Stem Cells for Glioblastoma Targeting and Therapy. Small 9, 4123-4129 (2013).

191. Muroski, M.E., et al. Controlled payload release by magnetic field triggered neural stem cell destruction for malignant glioma treatment. PloS one 11, e0145129 (2016).

192. Mooney, R., et al. Neural stem cell-mediated intratumoral delivery of gold nanorods improves photothermal therapy. ACS nano 8, 12450-12460 (2014).

193. Suvà, M.L., et al. Reconstructing and reprogramming the tumor-propagating potential of glioblastoma stem-like cells. Cell 157, 580-594 (2014).

194. Kamerkar, S., et al. Exosomes facilitate therapeutic targeting of oncogenic KRAS in pancreatic cancer. Nature 546, 498-503 (2017). 